\title{
LUT
}

University

\section{Impact of different forest densities on atmospheric boundary-layer development and wind-turbine wake}

Adedipe Taiwo A., Chaudhari Ashvinkumar, Kauranne Tuomo

This is a Author's accepted manuscript (AAM) version of a publication

published by John Wiley \& Sons

in Wind Energy

DOI: $10.1002 /$ we.2464

Copyright of the original publication: (c) 2020 John Wiley \& Sons, Ltd.

Please cite the publication as follows:

Adedipe, T.A., Chaudhari, A., Kauranne, T. (2020). Impact of different forest densities on atmospheric boundary-layer development and wind-turbine wake. Wind Energy. DOI: 10.1002/ we.2464

This is the peer reviewed version of the article, which has been published in final form at https:// doi.org/10.1002/we.2464. This article may be used for non-commercial purposes in accordance with Wiley Terms and Conditions for Use of Self-Archived Versions.

This is a parallel published version of an original publication. This version can differ from the original published article. 


\title{
Impact of different forest densities on atmospheric boundary-layer development and wind-turbine wake
}

\author{
Taiwo A. Adedipe*, Ashvinkumar Chaudhari, Tuomo Kauranne \\ Computational and Process Engineering, School of Engineering Science, \\ Lappeenranta-Lahti University of Technology, \\ P.O. Box 20, 53851 Lappeenranta, Finland.
}

January 17, 2020

\begin{abstract}
The aim of this work is to investigate the Atmospheric Boundary-Layer (ABL) flow and the wind-turbine wake over forests with varying Leaf-Area Densities (LAD). The forest LAD profile used in this study is based on a real forest site, Ryningsnäs, located in Sweden. The reference turbine used to model the wake is a welldocumented 5-MW turbine which is implemented in the simulations using an Actuator Line Model (ALM). All simulations are carried out with OpenFOAM using the Reynolds Averaged Navier-Stokes (RANS) approach. Twelve forest cases with Leaf Area Index (LAI) ranging from 0.42 to 8.5 are considered. Results show that the mean velocity decreases with increasing LAI within the forest canopy, but increases with LAI above the hub height. Meanwhile, the turbulent kinetic energy (TKE) varies non-monotonically with forest density. The TKE increases with forest density and reaches to its maximum at an average LAI of 1.70, afterwards, it decreases gradually as the density increases. It is also observed that the forest density has a clear role in the wake development and recovery. Comparisons between no-forest and forest cases show that the forest characteristics help in damping the added turbulence from the turbine. As a consequence, the forest with the highest upstream turbulence has the shortest wake downstream of the turbine.
\end{abstract}

keywords: Atmospheric Boundary-Layer; Actuator Line Model; Forest density; Turbulent kinetic energy; Wind-turbine wake; Wind-power.

\section{Introduction}

Forest areas have become an important resource to utilize wind power production with the use of windturbines. Recent research has shown that forested terrains generate lower wind speed and higher wind shear and turbulence intensity in the lower part of the Atmospheric Boundary-Layer (ABL) compared to flat terrains (i.e. without forest) ${ }^{1-3}$. This distorted flow has significant effects on the flow field as it increases the mechanical loads on turbine rotor and causes uncertainty in the power production ${ }^{1}$. Information regarding the flow disturbance induced by forests on wind-farms with different forest densities and how they affect wind energy potential will enable better selection of new wind-turbine types, wind-farm sites and enhance efficient production of wind power.

Series of numerical studies have been carried out on the simulations of wind flow over forest ${ }^{2 ; 4-10}$. In most of these studies, the forest canopy features have been explicitly represented in the simulation domain using the leaf area density (LAD). The inclusion of forest in simulations using LAD represents the effects of the trees by discrete drag elements exerted on the wind flow, and therefore, the approach accounts for the momentum exchange and the turbulence interaction within and above forest canopies.

The overall behaviour of turbulent flow over forest canopies has been explored over a range of forest densities ${ }^{5 ; 11 ; 12}$. Dupont and Brunet ${ }^{5}$ performed a numerical study over different types of canopies with leaf-area index (LAI) ranging from 1 to 5 . Novak et al. ${ }^{11}$ considered a uniform forest canopy at various densities created by removing whole trees in a regular pattern. Also, five different vegetation densities varying from an extremely sparse canopy to an extremely dense canopy were investigated by Huang et al. ${ }^{12}$. These studies clearly show the variability in turbulent flow over different forest canopies, and it has been confirmed that the typical features of turbulent flow over forest canopies become more pronounced as canopy density increases. However, an in-depth search and a closer look on existing literature reveals a number of gaps in connecting forest with wind energy.

There have been very few studies so far that investigated the effects of forests on a wind turbine. Nebenführ and Davidson investigated the ABL over a sparse and a dense forest, as well as over grasscovered flat terrain ${ }^{6}$. They studied the impact of two different forests and wind speed on the wind-turbine fatigue loads. Different turbine parameters were analysed and it was established that the presence of forest increases wind-turbine fatigue loads. Recently, Agafonova et al. ${ }^{13}$ investigated the effects of a forest canopy on ABL and wind-turbines in two identical cases; with and without forest. It was established that there are 
significant changes in the wake and turbulence structure over a forest cover compared to a non-forest (e.g field) scenario. The wake recovers earlier and faster in the case with forest than in the case without forest due to higher turbulent mixing involved in the ABL above forests. It was also observed that, the loss of available potential power due to forest is approximately $30 \%{ }^{14}$. This large power loss is presumed to occur due to an induced turbulence intensity as well as increased shear in the mean wind profile in the forested wind-farm ${ }^{15}$. Available findings in the literature suggest that more studies would be needed to address in detail the coupling between forest densities and wind-turbine wakes. Therefore, this study is aimed at investigating how different forest densities affect ABL development and a wind-turbine wake. In particular, the objectives are to study how the turbulent kinetic energy (TKE) profile behaves over different forest densities, and how the upstream TKE, generated due to the presence of forest, affects the wind-turbine wake characteristics.

The studied forest is a Scots pine (Pinus sylvestris) with densities varying from an extremely sparse $(\mathrm{LAI}=0.42)$ to an extremely dense $(\mathrm{LAI}=8.5)$ forest. Also, following previous studies ${ }^{2 ; 4 ; 6 ; 13}$, in which ABL flows over forest has been simulated, we assume each forest case to be homogeneous in the horizontal directions but the LAD is varying in the vertical direction. The numerical simulations have been carried out using the open-source code OpenFOAM ${ }^{16}$. Firstly, a 2-dimensional (2d) set up was considered with 12 different forest density cases as well as a case without forest to study the effect of various forest densities on ABL development, and in particular, the TKE generation due to change in density. Furthermore, based on the $2 \mathrm{~d}$ ABL flow results, 3 forest cases were selected to further study the effect of forest densities on the wake flow structure and its characteristics. The wind-turbine employed here is the well-documented large scale turbine NREL-5MW with the rotor diameter $126 \mathrm{~m}^{17}$, and the turbine wake is simulated by employing an Actuator Line Model (ALM) developed by Sorensen and Shen ${ }^{18}$.

The outline of the paper is as follows; Section 2 discusses the mathematical approaches used in this study; Section 3 presents the numerical set-ups of all simulations; Section 4 shows the validations of the present study; Section 5 presents all results and discussions; while the last section concludes the paper.

\section{Modelling Approaches}

The steady and unsteady state Reynold's Averaged Navier-Stokes (RANS) equations are utilized in the numerical simulations. The realizable $k-\epsilon$ turbulence model $^{19}$ is employed in all cases. The source terms used to model the forest canopy and turbine forces are described in the following subsections.

\subsection{Forest canopy model}

The forest canopy model used in this study is based on the drag force approach presented by Shaw and Schumann ${ }^{20}$. The forest drag force $\left(f_{i}\right)$ due to the forest canopy elements is given as;

$$
f_{i}=-C_{d} \alpha_{f}|U| U_{i}
$$

The model constant $C_{d}=0.15$ is the drag coefficient, $\alpha_{f}$ defines the LAD as a function of height, $|U|$ is the magnitude of the mean velocity vector and $U_{i}$ is the velocity in the $i$-direction.

The source term for the turbulent kinetice energy, $k$, is:

$$
f_{k}=\rho C_{d} \alpha_{f}|U|\left[\beta_{p}|U|^{2}-\beta_{d} k\right]
$$

The constant $\beta_{p}=0.17$ accounts for turbulence production due to the surface drag of canopy elements while $\beta_{d}=3.37$ accounts for the enhanced dissipation. The source term for the dissipation, $\epsilon$, is,

$$
f_{\epsilon}=\rho C_{d} \alpha_{f}|U| \epsilon\left[\frac{C_{\epsilon 4} \beta_{p}|U|^{2}}{k}-C_{\epsilon 5} \beta_{d}\right]
$$

Where $C_{\epsilon 4}=0.9$ and $C_{\epsilon 5}=0.9$ are model constants. Desmond et al. ${ }^{7}$ and some others have used this model and it has been verified as an accurate assessment of the porosity of the forest canopy ${ }^{2 ; 6 ; 21}$. The choice of values of the coefficients in the source terms equations are adopted from references ${ }^{6 ; 7 ; 9}$.

\section{$2.2 \quad$ Actuator line model}

The ALM, introduced by Sorensen and Shen ${ }^{18}$, is based on the three-dimensional Navier-Stokes equations combined with the blade element theory. In this model, the turbine blades are represented as actuator line elements on which the body forces are distributed. The actuator lines are discretized into discrete elements and the body forces on each of these small blade elements are determined by the lift and drag forces, the chord length, twist angle and two-dimensional airfoil data. The lift and drag forces acting on the blade element are defined, respectively as:

$$
\begin{aligned}
L & =\frac{1}{2} C_{L}(\alpha) \rho U_{r e l}^{2} C d \\
D & =\frac{1}{2} C_{D}(\alpha) \rho U_{r e l}^{2} C d
\end{aligned}
$$


where $C_{L}$ is the lift coefficient, $C_{D}$ is the drag coefficient, $\alpha$ is the local angle of attack, $\rho$ is the density, $U_{r e l}$ is the local wind speed, $C$ is the chord length and $d$ is the thickness of the blade element. Hence, the effects of these forces on the flow field are determined by the turbine force vector;

$$
S_{t}=\frac{\vec{L}+\vec{D}}{\epsilon^{2} \pi^{3 / 2}} \exp \left[-(r / \epsilon)^{2}\right]
$$

Where $\vec{L}$ and $\vec{D}$ are the lift and drag vector forces respectively, $r$ is the distance from the location of the actuator element center to the point where the body force is applied and $\epsilon$ is the projection width.

The ALM technique has been used by many researchers to model turbine wakes in numerical simulations $^{13 ; 22-29}$, and it has been compared with other existing turbine models such as the Actuator Disc Models $(\mathrm{ADM})$; rotating and non-rotating ${ }^{23 ; 24 ; 30 ; 31}$. It is established that the rotating ADM and the ALM better predicts the turbine wake, both in the near and far wake regions, and their predictions are always in good agreement with experimental results.

\section{Numerical set-ups}

This section is segmented into four subsections: the first sub-section describes the $2 \mathrm{~d}$ ABL flow simulations carried out for 12 different forest density cases. The second sub-section "3d turbine wake simulations" gives the details of the turbine wake simulations for three different forest cases selected in addition to the no-forest case. The third sub-section describes the mesh dependency study.

\subsection{2d ABL flow simulations}

There are twelve forest cases and one non-forest case considered in this division. The forest density is evaluated by the cumulative LAI defined as,

$$
L A I=\int_{0}^{h} \alpha_{f}(z) d z
$$

where $h$ is the forest height and $\alpha_{f}(z)$ is the LAD representing the amount of biomass of the canopy per unit volume and as a function of height. The LAD profile utilized in this study is the dense profile of the Scots Pine forest (Pinus silvestris) identical to a field experiment site at Ryningsnäs in south-eastern Sweden ${ }^{32 ; 33}$. This profile has been used and proved successful in a Large-Eddy Simulations (LES) study performed by Nebenführ and Davidson ${ }^{4 ; 6}$. Following Nebenführ and Davidson ${ }^{4}$, the forest height is $h=20 \mathrm{~m}$ and it is assumed to be horizontally homogeneous in the entire domain. The extracted LAD profile corresponds to a pine forest with $L A I \approx 4.25$, and this serves as the reference forest case from which the remaining LAD profiles are generated for other 11 forest cases. For analysis purposes, the forest cases are given generic names with respect to the density of the forest as presented in Table 1. Each forest LAD, from p1 to p9 increases by a multiplying factor of $10 \%$ of the reference forest (p10) while p11 is calculated by increasing the p10 LAD by half and p12 is by twice the LAD of p10. Figure 1 presents the LAD profiles of the various cases.

Table 1: Forest cases and their LAI values.

\begin{tabular}{|c|c|c|c|c|c|c|c|c|c|c|c|c|}
\hline Names & $\mathrm{p} 1$ & $\mathrm{p} 2$ & $\mathrm{p} 3$ & $\mathrm{p} 4$ & $\mathrm{p} 5$ & $\mathrm{p} 6$ & $\mathrm{p} 7$ & $\mathrm{p} 8$ & $\mathrm{p} 9$ & $\mathrm{p} 10$ & $\mathrm{p} 11$ & $\mathrm{p} 12$ \\
\hline LAI & 0.42 & 0.85 & 1.27 & 1.70 & 2.12 & 2.55 & 2.97 & 3.40 & 3.82 & 4.25 & 6.37 & 8.49 \\
\hline
\end{tabular}

The simulations are executed on a 2 d rectangular domain with an ABL height $L_{z}$ of $500 \mathrm{~m}$ and length of $3000 \mathrm{~m}$ in the stream-wise direction. The coordinates $(x y z)$ correspond to the stream-wise, span-wise and vertical directions, respectively. The domain was discretized into $600 \times 157$ cells and the global cell expansion ratio is 1 and 2.5 in the horizontal and vertical directions, respectively. This leads to a uniform grid resolution of $5 \mathrm{~m}$ in the horizontal direction while in the vertical direction, a $2 \mathrm{~m}$ grid size was maintained within the forest canopy height of $20 \mathrm{~m}$ and stretched from $2 \mathrm{~m}$ above the forest canopy to $5 \mathrm{~m}$ towards the top of the domain. In order to obtain comparable results in all cases, the mass flow rate are controlled such that it gives a velocity of $10 \mathrm{~ms}^{-1}$ at the reference height of $110 \mathrm{~m}$. The reference height represents the original hub height $90 \mathrm{~m}$ of the NREL $5 \mathrm{MW}$ turbine with an extension of $20 \mathrm{~m}$ forest height.

\subsubsection{Boundary conditions}

In this study, the recycling method called the mapped boundary condition (BC) at the inlet is employed in order to achieve a neutrally stratified equilibrium ABL profile. The mapped $\mathrm{BC}$ is applied at the inlet of the domain, where the flow variables, such as $U, k, \epsilon$, are recycled from the recycling plane, which is located $3 L_{z}$ ( $L_{z}$ is the ABL height) downstream of the inlet plane (see Figure 2). A number of studies ${ }^{14 ; 34 ; 35}$ recommend that the size of the computational domain should have a recycling distance of at least $3 L_{z}$ for mapping the flow, plus a minimum of an additional $1 L_{z}$ between the recycling plane and the area of interest. This area is called the buffer zone, which is meant to prevent any upstream influence from the area of interest in the 


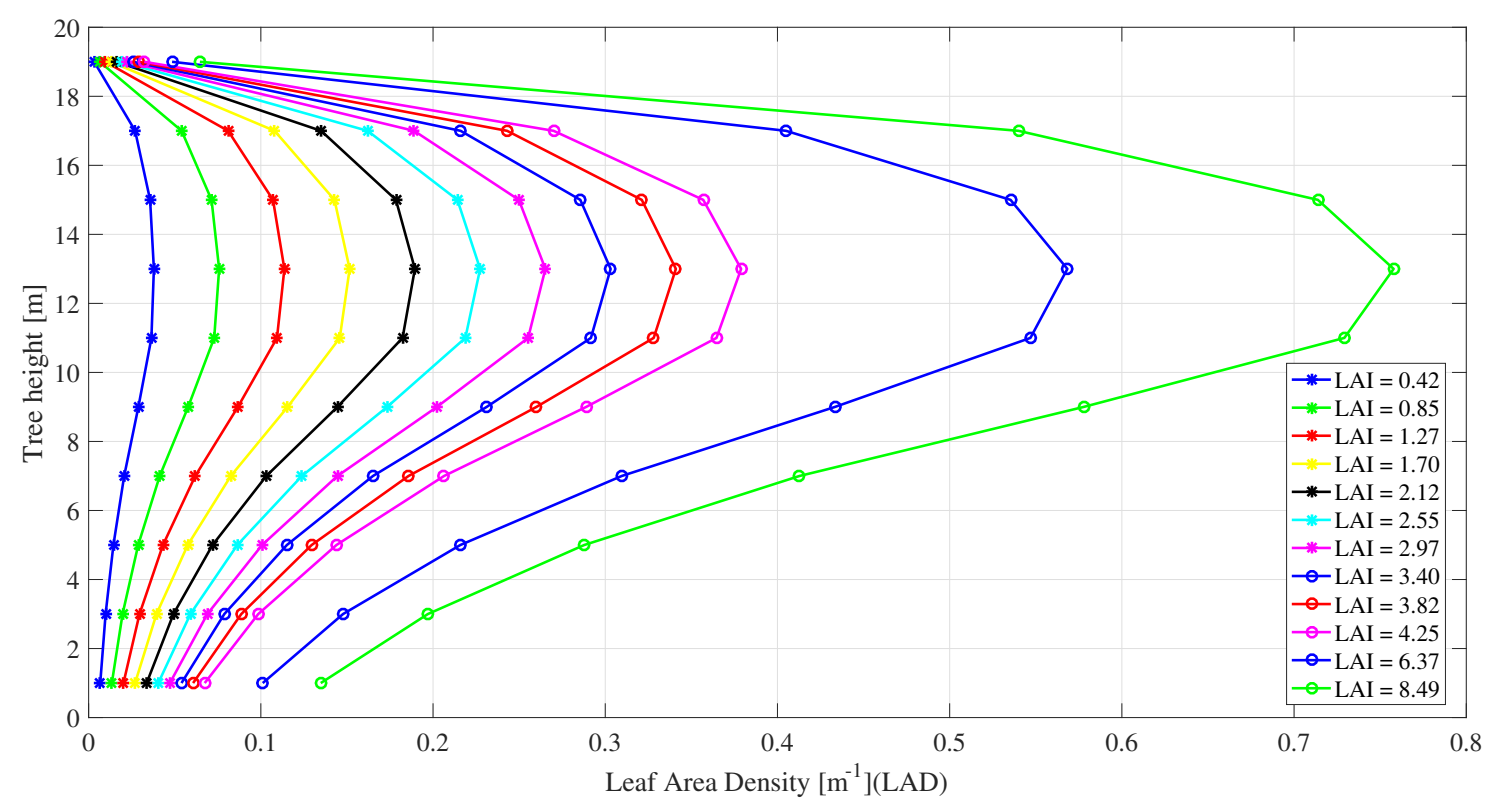

Figure 1: Leaf area density profiles of forest cases.

mapping region. The recycling process is repeated for each time step and eventually the flow profiles become fully developed naturally. During the entire simulation, together with recycling process, the flow is also fed naturally downstream (toward the main domain). The recycling method is described in details by BabaAhmadi and Tabor ${ }^{36}$, it has been used for atmospheric flow development with validation against full-scale experimental data ${ }^{34 ; 37 ; 38}$. Recently, it was also applied for ABL flow over forest canopy by Agafonova et $a l^{39}$, in which the technique successfully reproduced the fully developed upstream ABL over a forest site. The wall-function model based on the logarithmic $(\log )$ law over a rough surface, defined in Equation (8) is implemented on the terrain

$$
U(z)=\frac{U_{*}}{\kappa} \ln \left(\frac{z}{z_{0}}\right)
$$

where $U_{*}$ is the frictional velocity, $\kappa$ is the von Karman constant approximately $0.41, z$ is the vertical height and $z_{0}$ is the roughness length. The surface roughness length is set as $z_{0}=0.1 \mathrm{~m}$ and this effects only when the canopy density approaches to zero. The steady-state RANS solver in OpenFOAM is employed in the simulations. All simulations were converged with the solution convergence criteria set to $10^{-4}$.

\section{$3.23 \mathrm{~d}$ turbine wake simulations}

The domain used for the turbine wake simulations has dimensions $32 D, 6 D$, and $4 D$ in the stream-wise (x), span-wise (y) and vertical (z) directions, respectively, where $D=126 \mathrm{~m}$ is the diameter of the NREL 5 MW turbine. It is ensured that the domain is high enough such that the flow can no more be affected by the turbine effects, so also the span-wise distance. The stream-wise distance is, therefore, selected on the criteria that the stream-wise distance should be at least 7 times the vertical height of the computational domain when the recycling technique is in use ${ }^{35 ; 40}$. The domain is modelled in such a way that the turbine is located $17 D$ downstream of the inlet and $15 D$ upstream of the outlet. Figure 2 shows the schematic picture of the $3 \mathrm{~d}$ numerical domain with turbine location and all the dimensions.

Using the recycling $\mathrm{BC}$, the flow upstream is recycled at a distance of $12 \mathrm{D}$ from the inflow plane, and the buffer zone is $5 D$ between the recycling plane and the turbine. This recycling technique parameter enables the flow to be fully developed before the turbine. Using similar grid resolutions that have been established and used in earlier studies on flow over forest ${ }^{4 ; 8 ; 10}$, and forest canopy effects on turbine flow characteristics $^{6 ; 39}$, the computational domain is discretized into (806 x $\left.151 \times 158\right)$ grid-cells in $x, y$ and $z$ directions, respectively. A grid size of $5 \mathrm{~m}$ resolution in $x$ and $y$ directions is used, while, for the vertical direction, $2 \mathrm{~m}$ grid size is maintained within the forest canopy and stretched gradually to $5 \mathrm{~m}$ towards the top of the domain. The mesh is refined around the rotor area, horizontally from $1 D$ before the turbine to the outlet, and from above the forest to $1 D$ above the rotor tip in the vertical direction. This gives a $2.5 \mathrm{~m}$, approximately, $\Delta x=\Delta y=0.0198 D$ mesh resolution in the refinement (wake) region. A mesh dependency test proved that this resolution gives a mesh independent solution (see section 3.3). The same recycling method as described in subsection 3.1.1 is applied at the inlet boundary. The velocity at the hub height $\left(U_{H}\right)$ is adjusted to be $10 \mathrm{~ms}^{-1}$, and the Reynolds number $\left(R e_{D}\right)$, defined as a function of $D$ and $U_{H}$, is $8.9 \times 10^{7}$ 


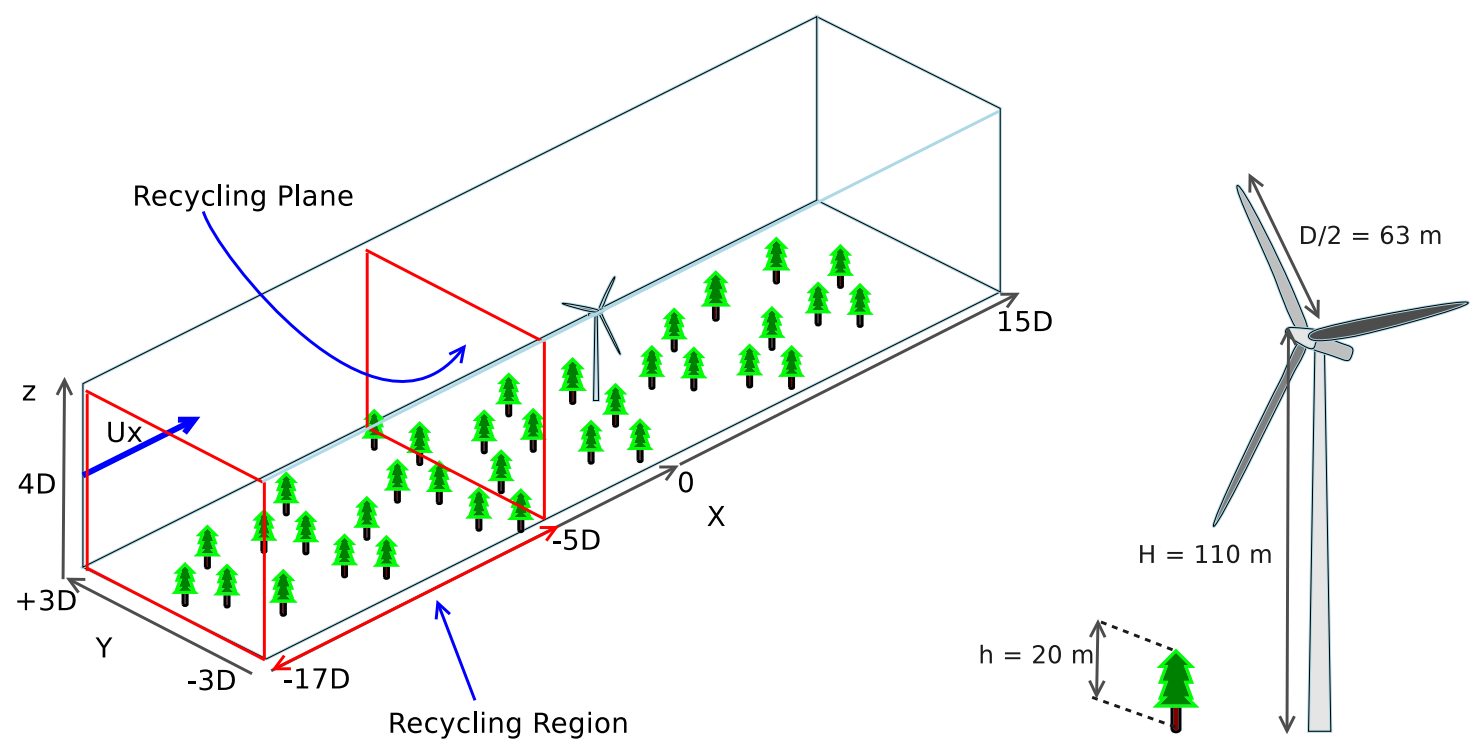

Figure 2: An overview of the computational domain showing the dimensions in terms of the rotor diameter $D$.

Unsteady RANS simulations of turbine wake over three forest cases and the no-forest case are carried out using the pimpleFoam solver ${ }^{16}$ of OpenFOAM. The pimpleFoam solver is based on SIMPLE and PISO (Pressure-Implicit with Splitting of Operators) algorithms and it's a standard solver for transient RANSbased turbulent flow simulations. The case without forest and three forest cases with LAI of $0.42,1.70$ and 4.25, respectively, are considered (cases p1, p4 and p10 from Table 1). The first two cases correspond to very sparse and sparse pine forests respectively, while $p 10$ is the reference forest (dense pine) described and used by Nebenfuhr and Davidson ${ }^{4}$. The aim is to investigate the turbulence intensity, and turbine-wake and characteristics in these cases. The backward, second order, and the second order Central Differencing (linear)schemes are used for time and spacial discretizations respectively. The $3 \mathrm{~d}$ simulations are run in two stages for a total run time of $6000 \mathrm{~s}$. In the first stage, the simulations are run for $5400 \mathrm{~s}$ of flow time in the absence of turbine in order to fully develop the flow fields over respective forest. In the second stage, the simulations are extended further (i.e. $5400 \mathrm{~s}$ onwards) for another $600 \mathrm{~s}$ of flow time with turbine included. In this way, the total simulated flow time is $6000 \mathrm{~s}$ which takes 13.75 hours of clock time in the reference forest case $p 10$, using 504 parallel processors on the Finnish supercomputer (Sisu) managed by CSC - IT Center for Science. The no-forest case takes $18 \%$ less clock-time. It was checked from the time series of the mean velocity and the mean TKE (not shown here) that the simulation time of $600 \mathrm{~s}$ was sufficient to have the statistically stationary mean flow quantities in the wake region. Therefore, the final results presented in Section 4 are taken at $6000 \mathrm{~s}$.

\subsection{Mesh dependency study}

The mesh dependency study is carried out by testing three different meshes on a full scale NREL 5 MW wind-turbine. The distribution of the grids is defined on the refinement region as $1.5 \mathrm{~m}, 2.5 \mathrm{~m}$ and $3.5 \mathrm{~m}$. This gives varying total number of cells, which are $8.4 \times 10^{7}, 2.2 \times 10^{7}$ and $9 \times 10^{6}$ respectively for $1.5 \mathrm{~m}$, $2.5 \mathrm{~m}$ and $3.5 \mathrm{~m}$ resolutions. Mesh resolution is of great importance in relation to the accuracy of RANS results, especially in turbine simulations. The grids need to be small enough to resolve the blade elements of the turbine rotor model (ALM) and the flow after it. Therefore, the quantitative effect of the different mesh resolutions on the wake region are investigated at different locations as presented in Figure 3 and 4 . Considering the mesh resolutions, we observe significant changes in the solution when the mesh resolution is increased from $2.5 \mathrm{~m}$ to $3.5 \mathrm{~m}$. However, by reducing the mesh resolution to $1.5 \mathrm{~m}$, the solution gives similar profiles as the $2.5 \mathrm{~m}$ mesh resolution results. This indicates a mesh independent solution at $2.5 \mathrm{~m}$ resolution.

Since there are no experimental data for the full scale NREL 5 MW turbine, the power output from the three cases owing to the three mesh resolutions are compared with the NREL 5 MW benchmark data presented in its documentation ${ }^{17}$. The NREL $5 \mathrm{MW}$ power output data is extracted for exactly $10 \mathrm{~ms}^{-1}$ velocity, the comparison of the power outputs and their respective percentage errors are shown in Table 2 . The $2.5 \mathrm{~m}$ mesh resolution has a reasonable agreement with the NREL $5 \mathrm{MW}$ power output, whereas the $1.5 \mathrm{~m}$ mesh resolution has a slightly better agreement with an over-prediction by $2.6 \%$. The $3.5 \mathrm{~m}$ mesh resolution give an under-prediction of $16 \%$.

Assessing the wake behaviour in the present RANS results due to different mesh resolutions, and also considering the power output in comparison with the reference turbine, it is computationally efficient to have a $2.5 \mathrm{~m}$ mesh resolution. It has also been demonstrated by $\mathrm{Su}$ et al ${ }^{10}$ that going below $2 \mathrm{~m}$ grid 

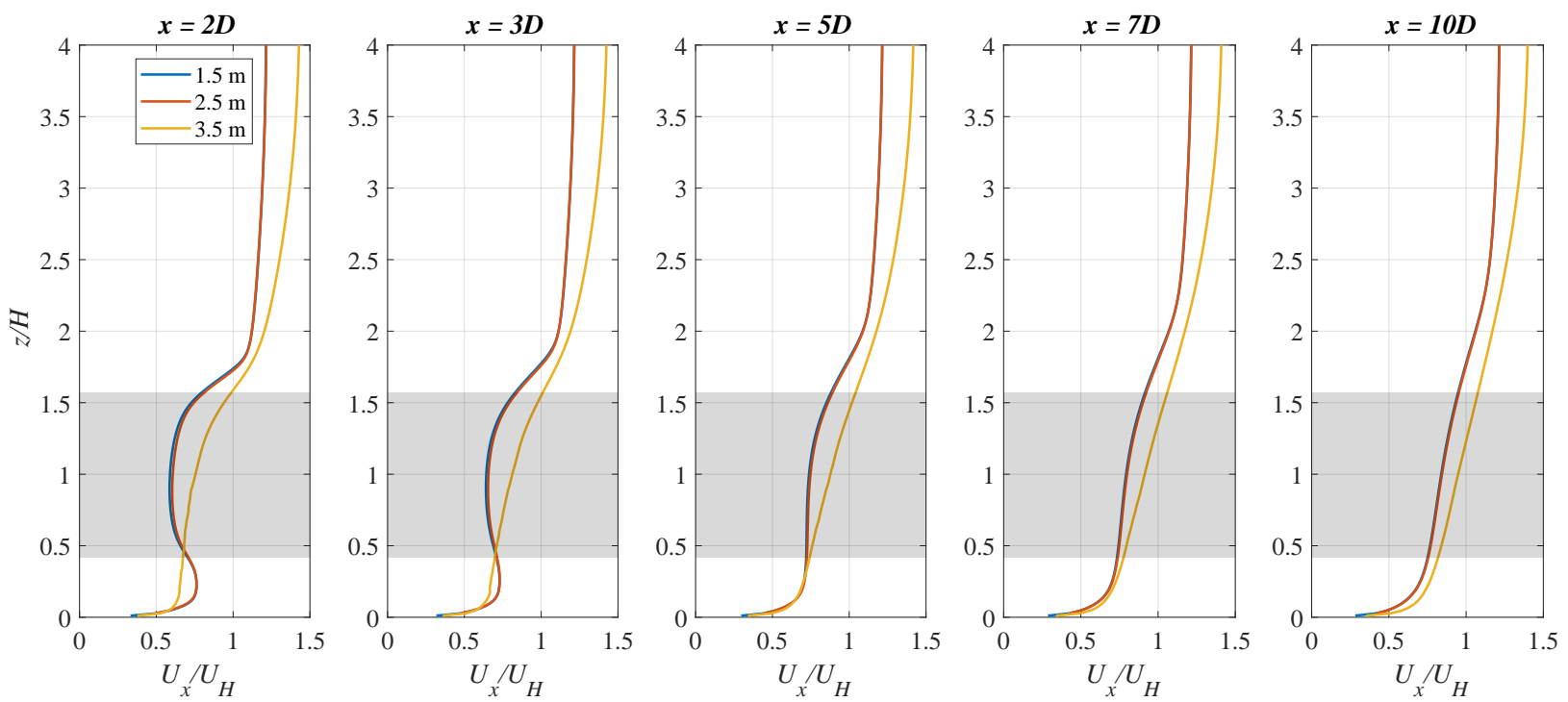

Figure 3: Vertical profiles of the normalized stream-wise velocity for different mesh resolutions at five different locations downstream of the turbine (The shaded region depicts the rotor area).
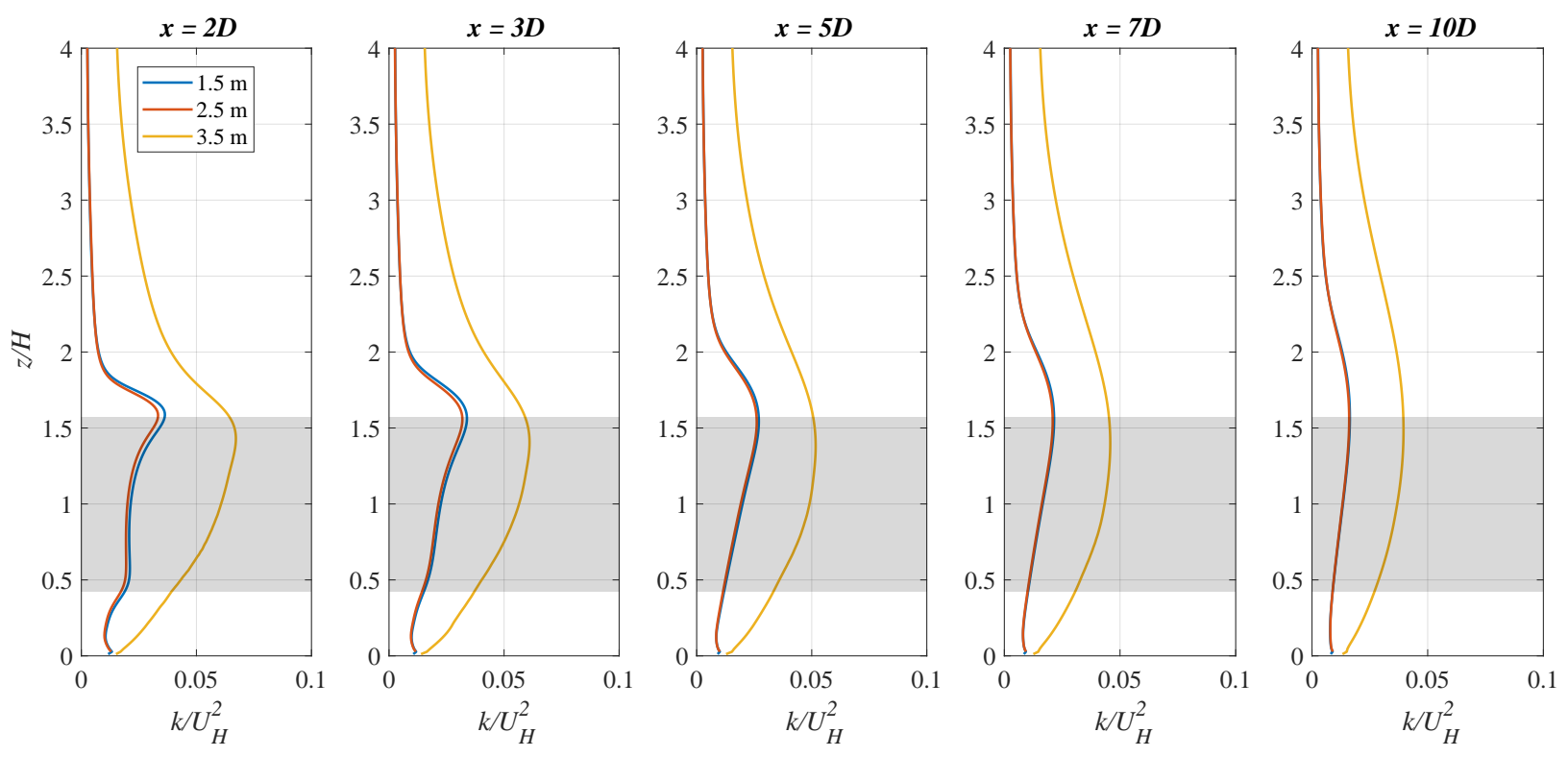

Figure 4: Vertical profiles of TKE for different mesh resolutions at five different locations downstream of the turbine (The shaded region depicts the rotor area).

resolution does not give any substantial difference in the results. In addition, similar grid resolutions have been used in turbine modelling of the NREL $5 \mathrm{MW}$ reference turbine with forest inclusion in previous studies $^{6 ; 39}$. The same resolution is, therefore, considered for all cases in this study. Moreover, similar grid resolutions have been used in turbine modelling of the NREL 5 MW reference turbine with forest inclusion in previous studies ${ }^{6 ; 39}$.

Table 2: Mesh independence test on NREL 5 MW

\begin{tabular}{|c|c|c|c|}
\hline Mesh resolution $(m)$ & 1.5 & 2.5 & 3.5 \\
\hline Number of cells & $83,994,624$ & $22,262,123$ & $9,274,176$ \\
\hline Processors & 960 & 504 & 216 \\
\hline CPU time (hours) & 30.5 & 11.3 & 9.8 \\
\hline Rotor power (MW) & 3.7634 & 3.5353 & 3.0746 \\
\hline NREL 5 MW & 3.6678 & 3.6678 & 3.6678 \\
\hline \% Error & +2.6 & -3.6 & -16.2 \\
\hline
\end{tabular}




\section{Validation of the numerical models with experimental re- sults}

This section is divided into two main sections, the first subsection validates the numerical simulations of the no-forest case before (for ABL development) and after the turbine (for wake modelling). The second subsection validates the simulation results of the $\mathrm{ABL}$ development over the forest canopy.

\subsection{Validation of wake simulations with wind tunnel measurements}

\subsubsection{CFD modelling for model wind turbine}

To validate the numerical modelling approach employed in this study, a small scale ABL flow over a miniature wind-turbine is simulated as no measurements are available for the virtual NREL 5 MW turbine. The results are then compared with the experimental measurements from the wind-tunnel study performed by Chamarro and Porté-Agel ${ }^{41}$. This experiment was carried out using a 3-blade GWS/EP-6030 33 model wind-turbine situated in the wind-tunnel of the Saint Anthony Falls Laboratory at the university of Minnesota in order to study the effect of ABL flow on wind-turbine wake characteristics. The turbine tower is $0.118 \mathrm{~m}$ high with a hub height of $0.125 \mathrm{~m}$ and a rotor diameter of $0.15 \mathrm{~m}$. The drag coefficient for the nacelle used is 0.85 . The experiments were performed with a boundary-layer depth of $L_{z} \approx 0.4 \mathrm{~m}$ and a freestream velocity of $2.5 \mathrm{~ms}^{-1}$. Explicit details about the experimental set-up are given by Chamarro and Porté-Agel ${ }^{41}$.

In this work, the boundary-layer depth is taken to be $L_{z}=0.45 \mathrm{~m}$, which is equivalent to $3 D_{w t}$ in terms of the rotor diameter $D_{w t}=0.15 \mathrm{~m}$. The stream-wise distance is therefore taken to be $36 D_{w t}$, where the first $3 L_{z}=9 D_{w t}$ is for mapping the flow, the next $4 L_{z}=12 D_{w t}$ is considered as the buffer zone and the remaining part of the domain, $5 L_{z}=15 D_{w t}$, is the area of interest where the turbine is located and the span-wise distance is $6 D_{w t}$. The hub height $(\mathrm{H})$ is $0.125 \mathrm{~m}$ and the velocity at the hub $\left(U_{H}\right)$ is $2.2 \mathrm{~m} \mathrm{~s}^{-} 1$. The domain is first discretized into $(518,58,64)$ hexahedron cells in the $(x, y, z)$ directions representing the stream-wise, span-wise and vertical directions respectively. The mesh is afterwards refined from $-D_{w t} / 2$ to $D_{w t} / 2$ around the rotor in the span-wise and vertical directions, and stream-wise from $1 D_{w t}$ before the turbine to the outlet. This finally gives a mesh resolution of $\Delta x=\Delta y=\Delta z=0.0347 D_{w t}$ in the wake region. Comparison of the wind-tunnel mesh resolution against the full scale simulations in terms of their respective rotor diameter shows a finer mesh resolution $(\Delta x=\Delta y=0.0198 D)$ in the full scale simulations. The log-law for the mean flow is fitted using a frictional velocity of $0.13 \mathrm{~ms}^{-1}$ and roughness length $z_{0}$ is taken as $0.0001 \mathrm{~m}$. The Reynolds number $R e_{D_{w} t}$ is $2.6 \times 10^{4}$ based on the rotor diameter and the velocity at the hub.

Regarding the airfoil type to be used, the airfoil type was not specified in the corresponding experimental report, but the same case has been numerically examined by Wu and Porté-Agel ${ }^{42}$, where they considered the rotor blade as a flat plate, hence, the flat plate airfoil is employed in this study. The tip-speed ratio is 4 and this leads to a rotor angular velocity of $1117 \mathrm{rpm}$.

The simulations are based on the unsteady RANS approach using the pimpleFoam solver of OpenFOAM, and are executed in two stages for a total run time of $25 \mathrm{~s}$. At first, the simulations are run for $20 \mathrm{~s}$ of flow time to develop the inflow ABL profile, and afterwards for another $5 \mathrm{~s}$ of flow time with turbine included. The total $25 \mathrm{~s}$ of simulated flow time takes 4 hours of clock time using 48 parallel processors. The total simulation flow time is found sufficient to have the statistically stationary mean flow quantities before the turbine and in the wake region.

\subsubsection{Wake modelling}

The numerical results from the miniature wind-turbine simulations are presented against the experimental measurements from Chamarro and Porté-Agel ${ }^{41}$ in this section. The experimental data are digitally extracted from $\mathrm{Wu}$ and Porté-Agel ${ }^{42}$, and comparisons are made for the inflow and wake profiles of the mean flow and turbulence intensity. The inflow profiles for velocity and the turbulence intensity are presented in Figure 5. A maximum discrepancy of $7 \%$ is observed at $z / H \approx 0.1$ between the simulation result and the experimental data for the mean flow. Meanwhile, the simulation under-predicts the turbulence intensity below $z / H=1.2$ with a maximum discrepancy of $10 \%$ observed at $z / H \approx 0.08$.

Figure 6 shows the stream-wise velocity profiles at different stream-wise locations downstream of the turbine, while the turbulence intensity at the same locations is presented in Figure 7. Generally, from Figure 6 , the simulation underestimates the wind speed almost everywhere in the wake region with a maximum error of $43 \%$ at the turbine height. However, these deviations diminishes gradually downstream with a negligible error at $10 D_{w t}$ location. From Figure 7, the maximum discrepancy in the turbulence intensity is observed at the $2 D_{w t}$ location. The present RANS results show a maximum discrepancy of about $32 \%$ at $z / H \approx 0.4$, nonetheless, the deviations reduces and the error becomes negligible as the wake-flow recovers downstream. 

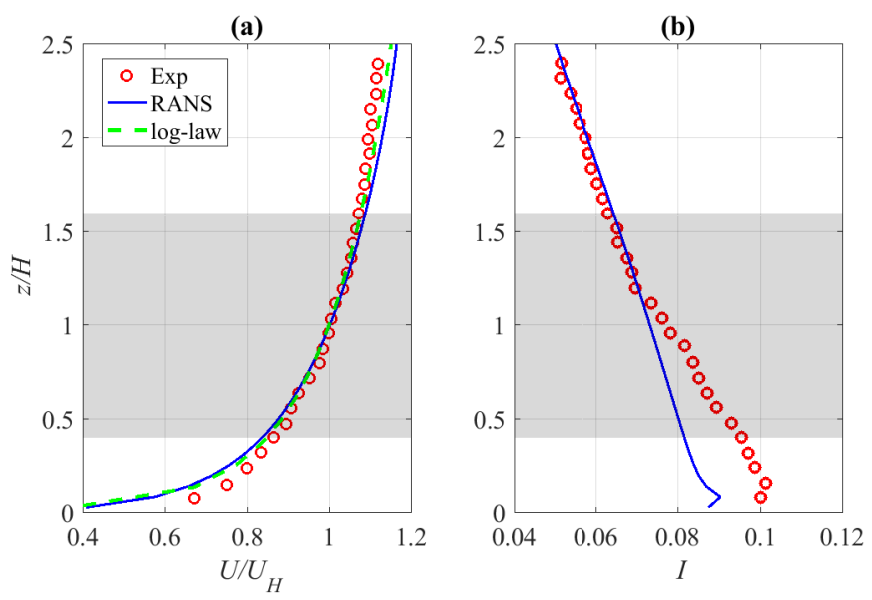

Figure 5: Inflow profiles from the present RANS result (blue line) against experimental data (red circles) ${ }^{42}$. The left plot shows the stream-wise velocity and the right plot shows the turbulence intensity.
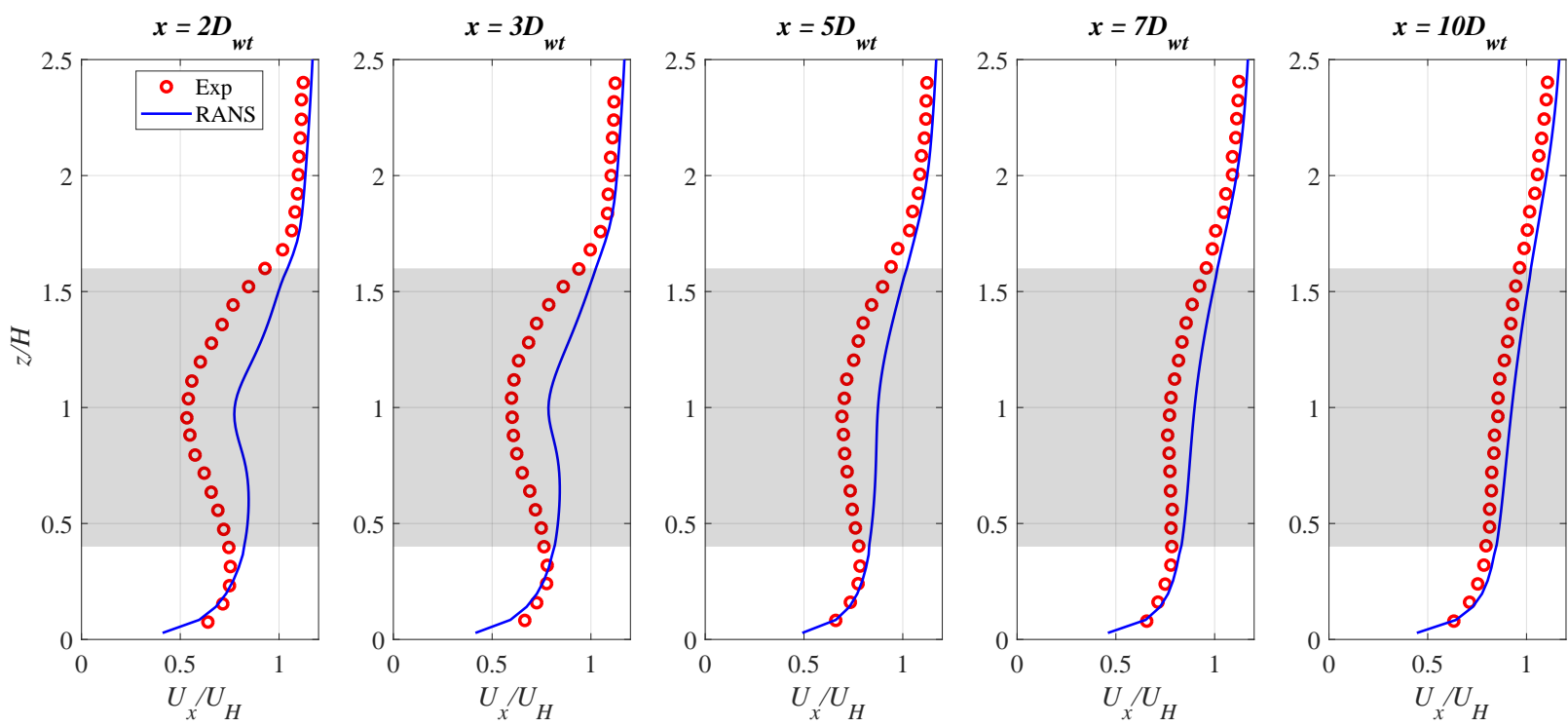

Figure 6: Vertical profiles of the normalized stream-wise velocity from the present RANS result (blue line) against experimental data (red circles) ${ }^{42}$ at five different locations downstream of the turbine.

\subsection{Validation of forest canopy model with field measurements}

The results from the reference forest case $(p 10)$ is validated against the experimental data from the Vindforsk III project V-312 ${ }^{32 ; 33}$. This experiment was carried out at Ryningsnäs in Sweden between November 2010 and February 2012. The forested landscape predominantly consists of Scots pine (Pinus silvestris). In the report $^{32 ; 33}$, the analysis and interpretation of measurements from a $138-\mathrm{m}$ tall tower located in the forest was presented, the mean canopy height $(h)$ near the mast was approximately $20 \mathrm{~m}$. Wind measurements were taken at different levels above the forest canopy ranging from $z / h=2$ to 7 with a sampling frequency of $20 \mathrm{~Hz}$ using the Sonic anemometers. In the experiment ${ }^{32 ; 33}$, six different classes of atmospheric conditions ranging from extremely unstable conditions to very stable conditions were considered. Among all the stability cases, the data from the neutral case are considered here for the validation of the simulations.

Figure 8 shows the normalized results of the TKE and mean wind speed from the present simulations in comparison with the experimental data ${ }^{32 ; 33}$. The results are taken exactly at the turbine location in the $2 \mathrm{~d}$ simulations (without turbine), although the vertical profiles are the same at every stream-wise and span-wise locations, as the forest is horizontally homogeneous. The simulation underestimates the TKE at every point with a maximum disparity of $7 \%$ and increasingly underestimates the wind speed above $z / h=3$ with a maximum disparity of $9 \%$. Overall, these results are considered reliable, seeing that all the data points falls within the confidence interval estimate from the statistics of the experimental data.

Since the aim of this research work is to investigate the ABL and turbine wake behaviour in forests with the same characteristics in terms of tree height, shape and type, all forest cases are, therefore, the replica of the one presented in this validation. The LAD of the reference case (p10) used in the validation is scaled with a factor of $10 \%$ in cases $\mathrm{p} 1$ to $\mathrm{p} 9$, increased by half in case p11 and doubled in case p12. In this respect, 

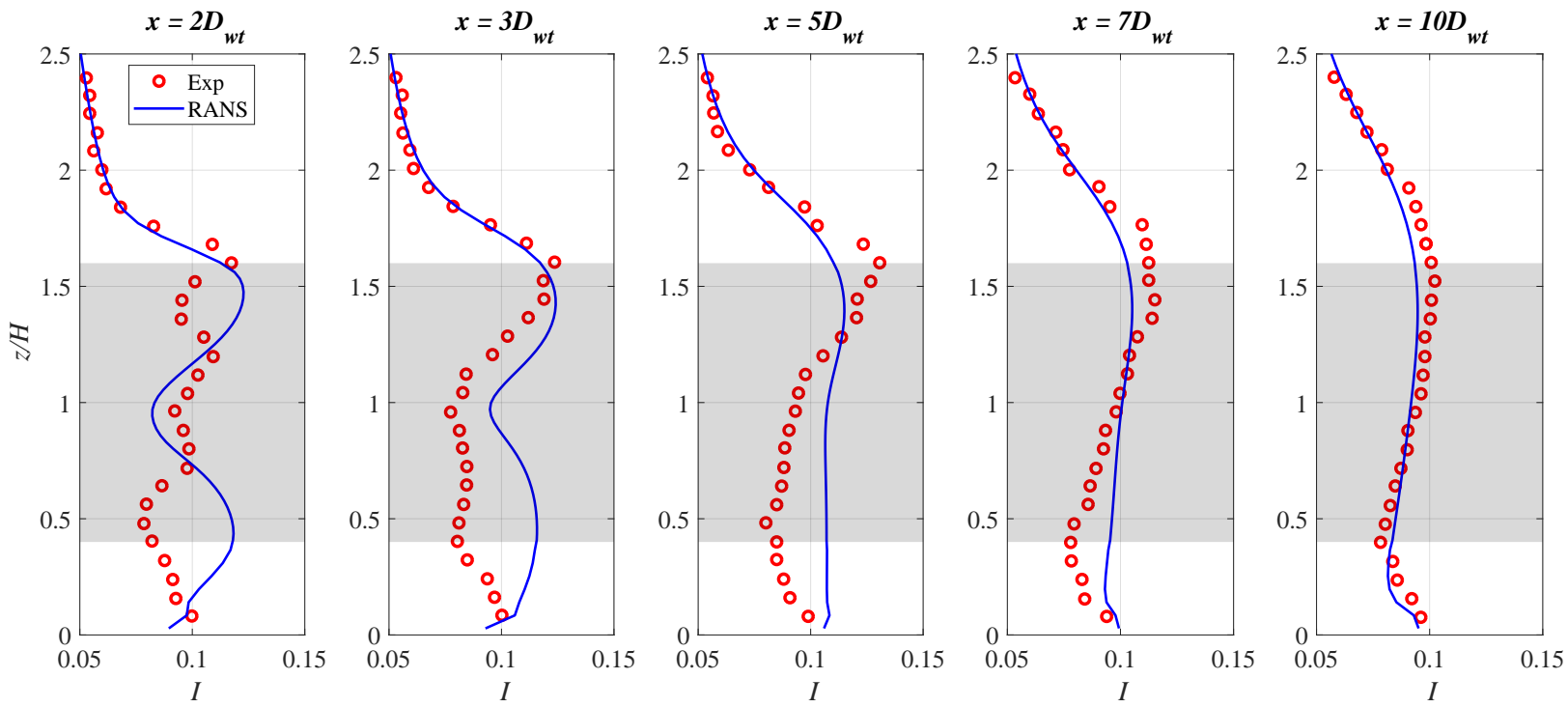

Figure 7: Vertical profiles of the normalized turbulence intensity from the present RANS result (blue line) against experimental data (red circles) ${ }^{42}$ at five different locations downstream of the turbine.

this validation is considered valid for all the forest cases.

(a)

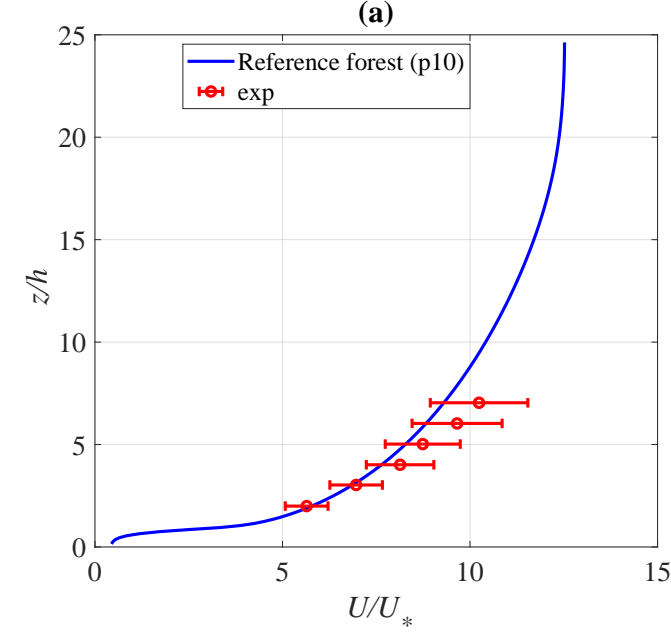

(b)

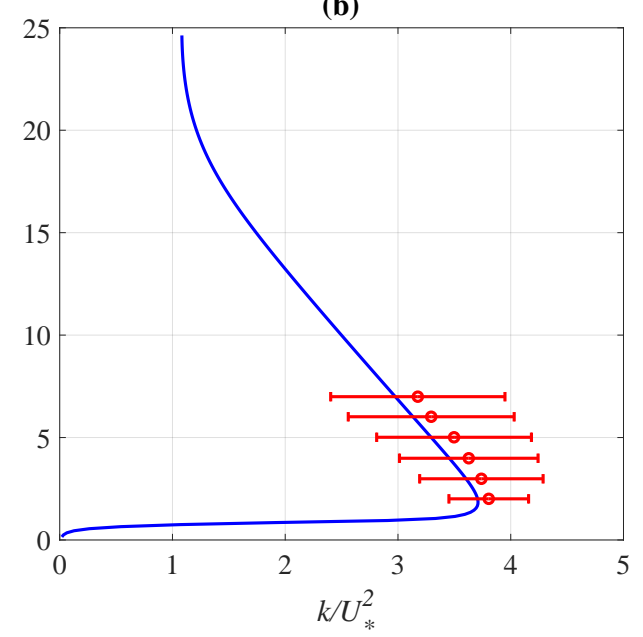

Figure 8: Vertical profiles of mean stream-wise wind velocity (left) and TKE (right) from the reference pine case (p10); Solid line represent the present numerical results while circles shows the experimental data ${ }^{32 ; 33}$ 


\section{Results and discussions}

This section is divided as follows; Subsection 5.1 shows the $2 \mathrm{~d}$ simulations results and discusses how different LAIs change the ABL profiles, and, Subsection 5.2 reports the results from turbine simulations and focuses on the wake effects.

\subsection{ABL flow simulations results}

The vertical profiles of the mean velocity and TKE for all cases are presented in Figure 9, where the vertical height is normalized by the tree height, $h=20 \mathrm{~m}$, and the velocity is normalized by the friction velocity. From Figure 9, comparing the no-forest and forest cases, there is a stronger wind shear and much higher TKE in the forest cases. The presence of forest is responsible for the higher turbulence above the canopy and it accounts for approximately $500 \%$ TKE increment in the forest cases (considering the reference forest p10) when compared to the no-forest case at all heights. Furthermore, it is observed from the mean flow in Figure 9(a) that the velocity decreases with increasing LAI within the forest canopy. This shows that the higher the forest density, the lower the wind speed within forest canopy. Above the hub height, the velocity increases with LAI only from $\mathrm{p} 1$ to $\mathrm{p} 4$, the velocity profiles in cases $\mathrm{p} 5-\mathrm{p} 12$ are the same above the forest area, therefore density does not affect the mean wind speed in forests with $L A I>2.5$.
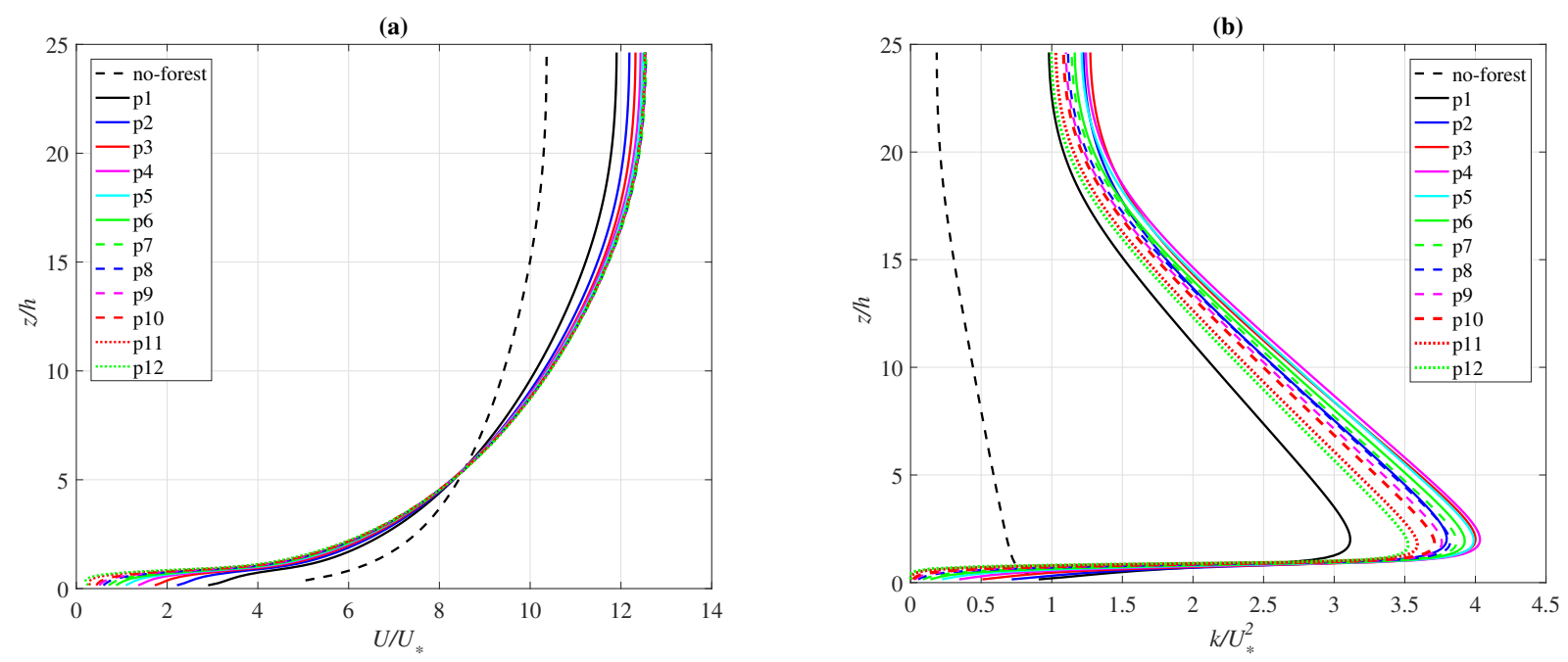

Figure 9: Vertical profiles of mean stream-wise wind velocity and TKE.

Generally, it is observed here that the TKE increases as the forest density increases and reaches to its maximum level with LAI approximately between 1.27 and 2.12 (p3, p4 and p5), it then gradually diminishes towards denser forests. Observing TKE at varying heights, the typical behaviour of TKE in atmospheric flow within and above a forest canopy is clearly seen in Figure 9(b). TKE is minimal in the lower part of the forest and increases from the forest-top to its peak at the upper layer above the forest canopy, it then diminishes gradually with height, but never to zero. This is because the forest canopy elements exert a drag force on the atmospheric flow which consequently produces turbulence above the forest ${ }^{43}$.

Furthermore, the plot of TKE against LAI for all cases is presented in Figure 10 to observe the actual relation between TKE production and LAI in an increasing density of forest. The TKE is normalized with the TKE from the no-forest case in order to better compare the ratio of TKE changes in forest cases directly with the no-forest case. The results indicate a non-monotonic relation between the forest densities and TKE. The physics of flow can be related to the permeability level of the forest canopies, since all forest cases have the same characteristics (tree height, type and shape), except the varying local density which defines the porosity or permeability of each forest. The permeability of the forests decreases as the LAI increases, but the behaviour of LAI versus TKE does not linearly correlate as a function of the permeability levels. From p1 to p4, the decreasing permeability (i.e. increasing LAI) triggers the vertical flow fluctuations, and they further become more frequent with increment in density, thus producing an increasing TKE above the canopy. But from p5 to p12, an opposite behaviour is observed, where the vertical flow movement over the canopy begin to decrease as the permeability continuously decreases. This in-turn produces a gradually decreasing TKE above the canopy with increasing LAI. In all the forest cases, p4 happens to have the most appropriate permeability which allows the highest vertical flow movements within the forest canopy, and hence, the highest TKE production above the canopy. 


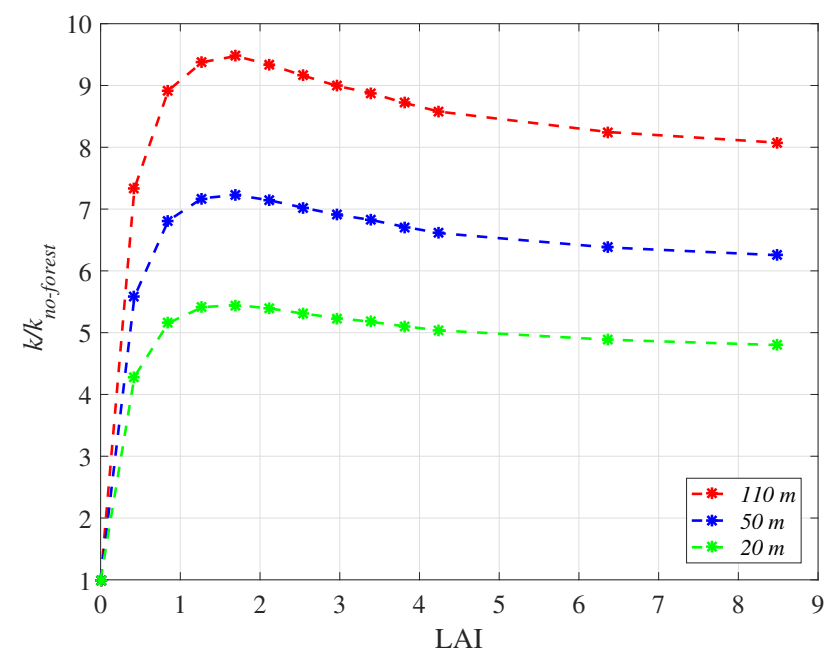

Figure 10: Normalized TKE results against LAI values taken at different heights for all cases including no-forest case.

\subsection{Turbine wake simulations results}

From the ABL flow simulation results, three forest cases (p1, p4 and p10) with clear distinctions in the TKE behaviour with respect to LAI are selected plus the no-forest case to investigate the impact of forest density on wind-turbine wake. To study the wake and its characteristics with respect to various forest densities, the velocity-deficit $(\Delta U)$ and TKE-increase $(\Delta k)$ are presented. $\Delta U$ and $\Delta k$ are calculated using the following expressions,

$$
\begin{gathered}
\Delta U=\frac{\left|U_{\text {inflow }}-U_{\text {wake }}\right|}{U_{\text {inflow }}} \\
\Delta k=\frac{\left|k_{\text {inflow }}-k_{\text {wake }}\right|}{k_{\text {inflow }}}
\end{gathered}
$$

Where $U_{\text {inflow }}$ and $k_{\text {inflow }}$ are the upstream profiles for velocity and TKE respectively, while $U_{\text {wake }}$ and $k_{w a k e}$ are the velocity and TKE profiles downstream of the turbine respectively. Results are taken at five stream-wise locations at the distance of $0 D, 1 D, 3 D, 5 D$ and $7 D$ downstream of the turbine, the vertical height is normalized by the hub height $(H)$ and the horizontal distance by radius $(R)$.

Figure 11 shows the contours of the velocity-deficit downstream from a horizontal plane at the hub height in all cases while Figure 12 shows the same contours on vertical planes extracted at few horizontal locations. It is generally observed that the wakes are much weaker and shorter with forest compared to no-forest. The no-forest case has a larger wake development, and the effect of the turbine exactly at the turbine location is more apparent without forest than with forest. Among all forest cases, p1 which is the most sparse has an insignificant difference with p10 in the wake region. p4 happens to have the shortest wake in all the forest cases.

In addition, the profiles of the velocity-deficit and TKE increase are presented to further investigate the wake structure. Figure 13 shows the vertical profiles of velocity-deficit for all cases. At $x=0 D$, it is noted that above the hub, the velocity-deficit increases in order of no-forest $\rightarrow \mathrm{p} 1 \rightarrow \mathrm{p} 10 \rightarrow \mathrm{p} 4$ and behave oppositely below the hub. This is found in the relation to the order of their upstream TKE as presented in Figure 9(b). A similar order is sustained on the next location $(x=1 D)$, but this time around, p1 and p10 almost overlap. For the rest of the locations, p1 and p10 are similar above the hub, but, below the hub, there is a shift between them such that the deficit is slightly higher in p1 compared to that in p10 at $1 D$.

For the stream-wise locations $x \geq 5 D, \mathrm{p} 1$ and p10 have almost the same wake behaviour, as the velocitydeficit difference between them is negligible (i.e. absolute error) despite the fact that there is a significant difference in their TKE levels. Overall, except at the turbine location $(x=0 D), \mathrm{p} 4$ has the weakest wake, while the no-forest case has the strongest wake and this is maintained at all locations. This is due to the fact that higher TKE level means higher turbulent mixing and that the flow recovery is faster leading to weaker wake. With respect to the no-forest case, the wake at $x=3 D$ is about $47 \%$ weaker in $\mathrm{p} 4$, and approximately $39 \%$ weaker in $\mathrm{p} 1$ and p10. Also, it is discovered that forest helps to destroy the near-wake effects sooner, as the turbine effect is still visible in the no-forest profile at $x=1 D$ which is no more visible in the forest cases at the same location.

Figure 14 shows the horizontal profiles of velocity-deficit and a similar phenomenon as seen in Figure 13 is exhibited, except at $x=0 D$ where the flow before and after the rotor does not follow a particular trend. It can also be observed that wakes are wider with forest. The peak values occur exactly along the center $(y / R=0)$ and a Gaussian shape of the velocity-deficit is found at all locations downstream of $x=1 D$. 


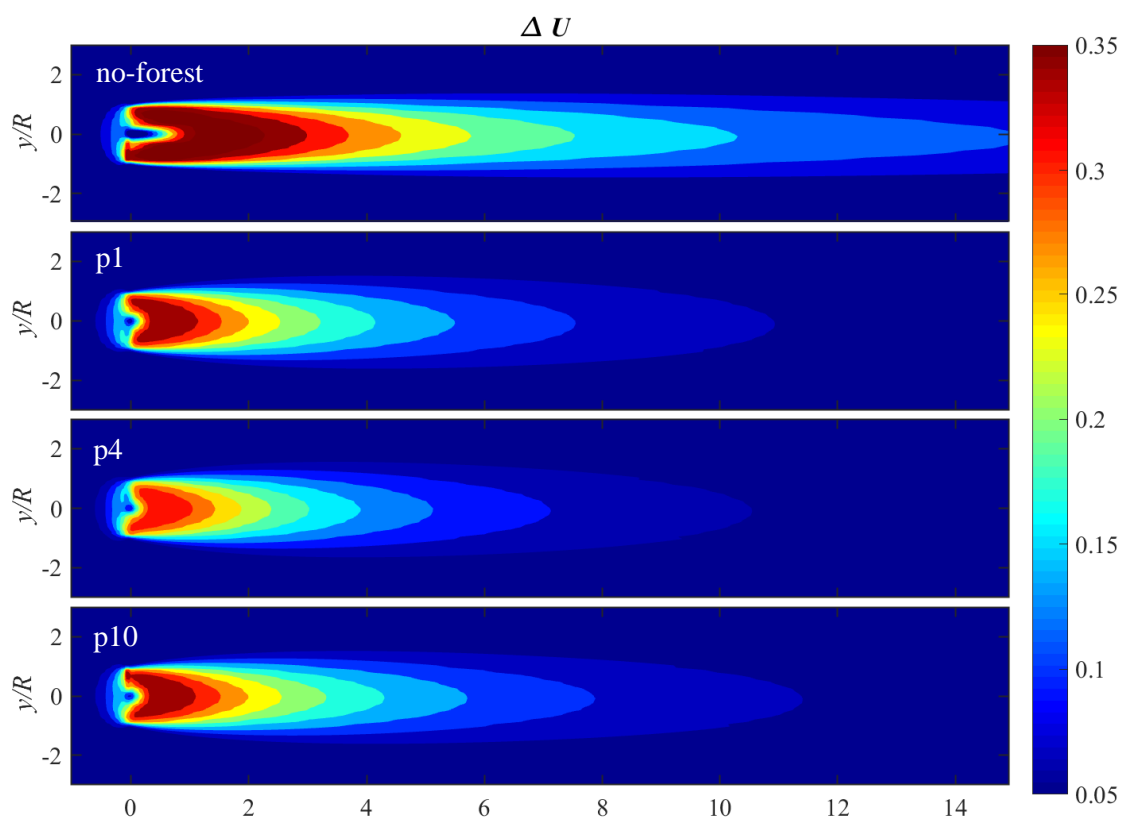

Figure 11: Velocity-deficit $(\Delta U)$ contours on horizontal planes at hub height.
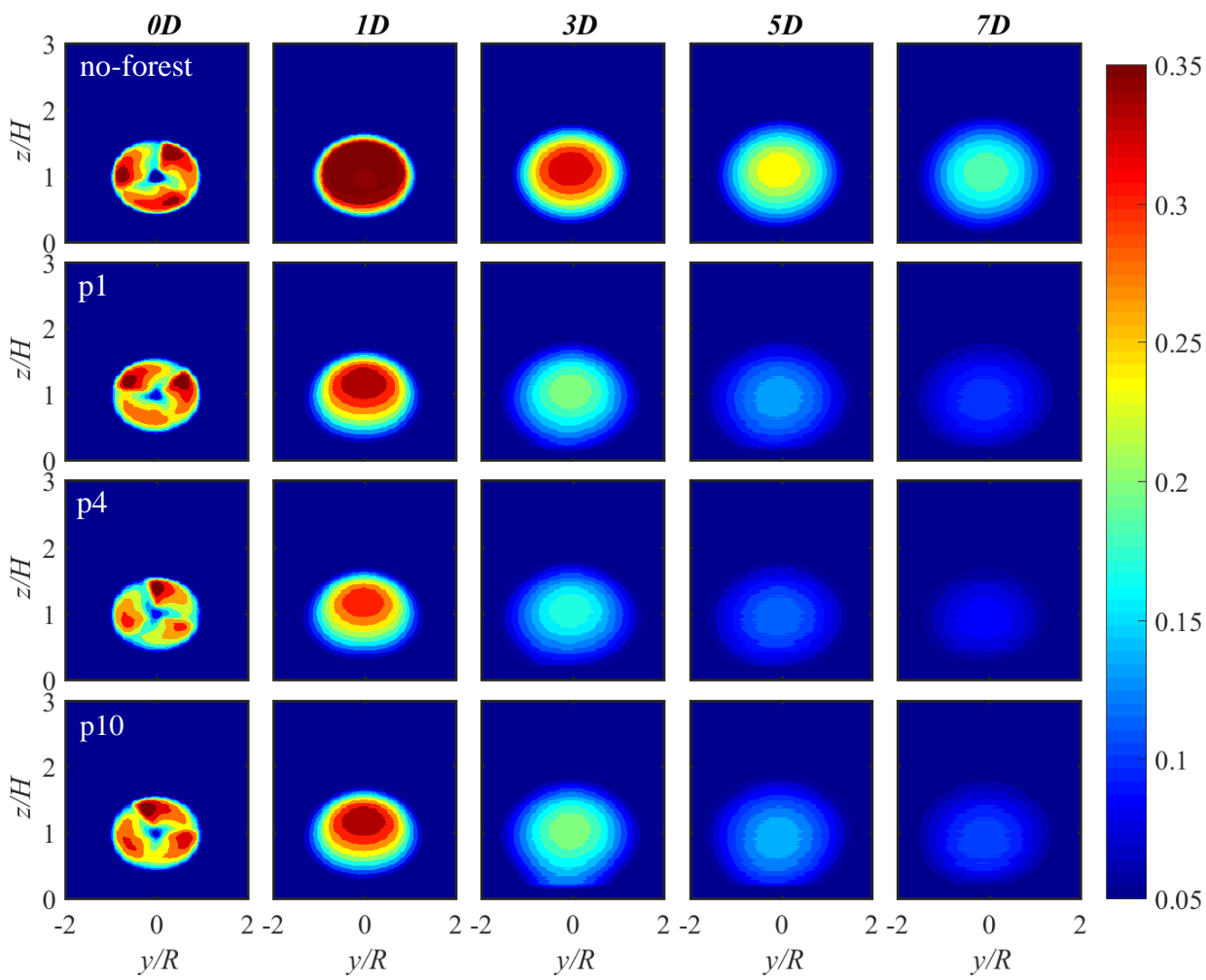

$y / R$

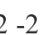

0
$y / R$

Figure 12: Velocity-deficit $(\Delta U)$ contours on vertical planes at five different locations downstream of turbine.

Figures 15 and 16 show the TKE increase contours from the horizontal and vertical planes respectively. Among the three forest cases, $\mathrm{p} 4$ which has the highest upstream TKE has the shortest wake and the fastest wake recovery, p10 with a lower upstream TKE has longer wake, while p1 that has the least upstream TKE has the longest wake. Generally, the forest cases exhibit more rapid wake recovery than the no-forest case.

The vertical profiles of the TKE-increase are presented in Figure 17. The maximum TKE-increase for each location is found to occur at the upper tip of the turbine. The highest magnitude of the TKE-increase 

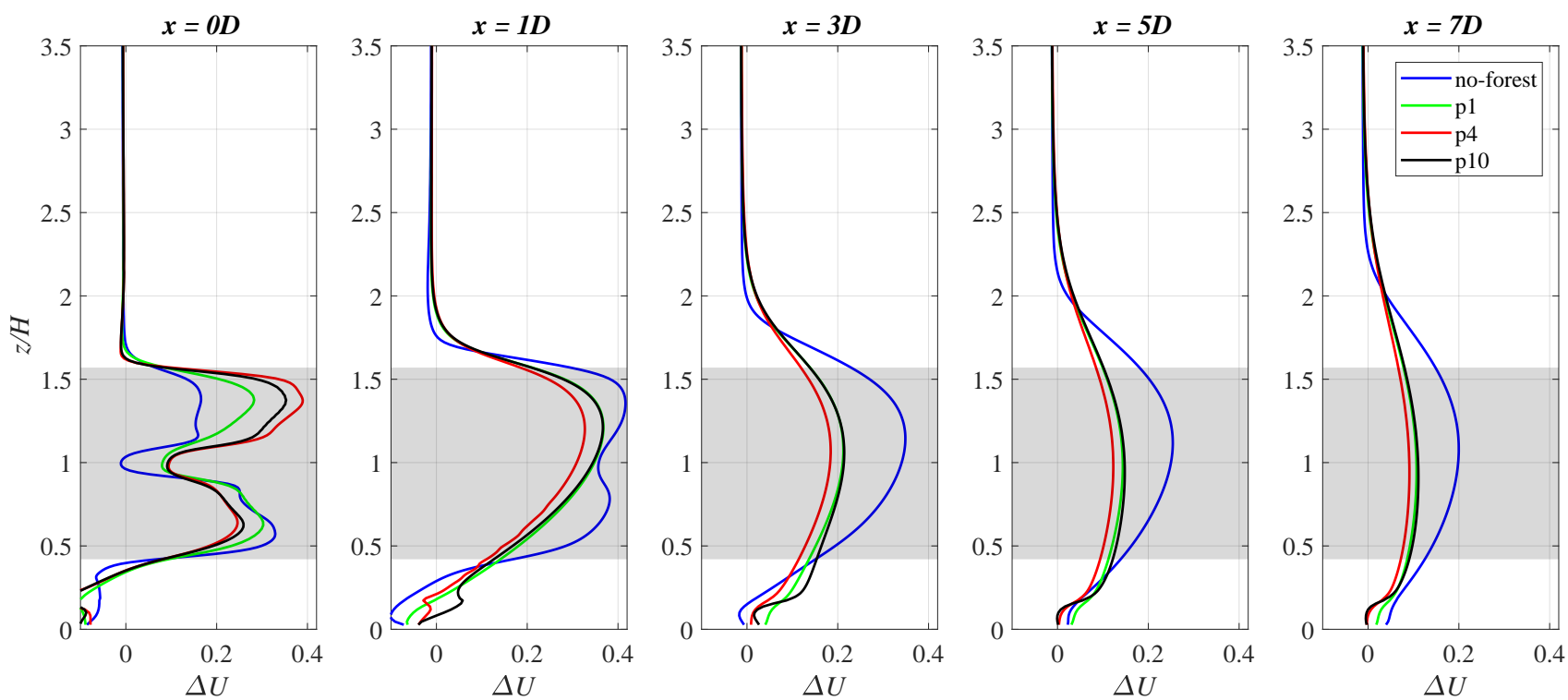

Figure 13: Vertical profiles of the velocity-deficits $(\Delta U)$ at five different locations downstream of the turbine (The shaded region depicts the rotor area).
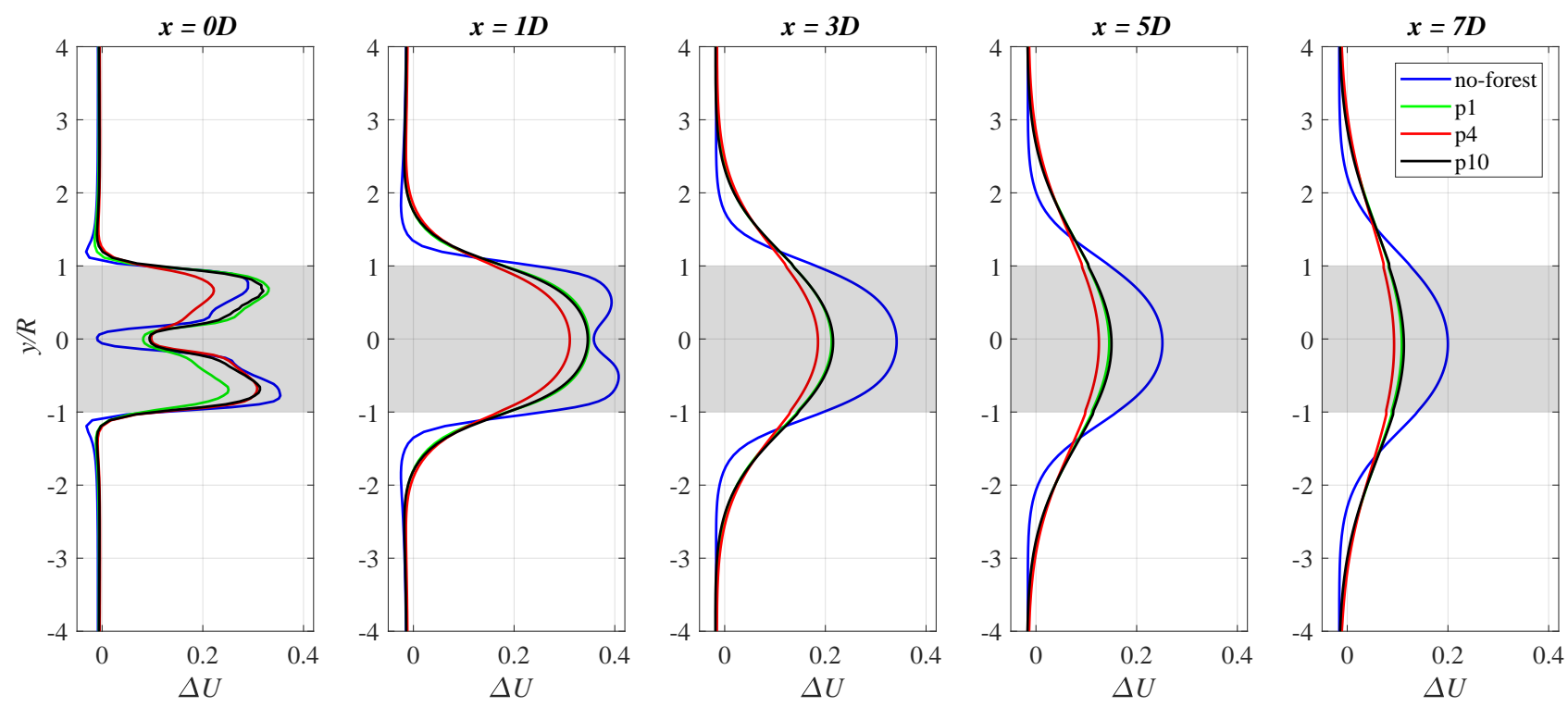

Figure 14: Horizontal profiles of the velocity-deficits $(\Delta U)$ at five different locations downstream of the turbine (The shaded region depicts the rotor area).

in the no-forest case is found at $x=3 D$. Such turbulence behaviour was also reported in an LES and a wind-tunnel study by Porté-Agel et al. ${ }^{44}$. Among all forest cases, the highest TKE-increase is found at $x=1 D$ in $\mathrm{p} 1$. This further indicates that the TKE-increase (due to wake) vanishes faster in the forest cases. These high magnitudes in TKE-increase (in all cases) diminish gradually away from the turbine and thus, the flow recovers its ambient condition.

From Figure 18, it is observed that in the no-forest case, the maximum TKE-increase values along the span-wise direction appear at the rotor tips for all the locations $(0 D-7 D)$, while in the forest cases, such peaks are visible only for the first two locations $(x=0 D$ and $x=1 D)$ and then the profiles change with weaker peaks concentrated in the center $(y / R=0)$. This indicates a huge damping of the TKE-increase in the forest cases, which is highly beneficial for the next turbine. At $x=1 D$, with respect to the corresponding upstream TKE level, the TKE value in the no-forest case is about $270 \%$ higher, while such statistics from the forest cases are $63 \%, 50 \%$ and $42 \%$ in p1, p10 and p4 cases, respectively. Hence, it can be deduced that the forest density (and therefore the upstream TKE level) is playing a significant role here as the smallest TKE-increase is found in the p4 case, which has the strongest upstream TKE. However, considering at $x=7 \mathrm{D}$ location, the impact of forest density is much milder, as the maximum absolute difference among the forest cases is only $7 \%$. 


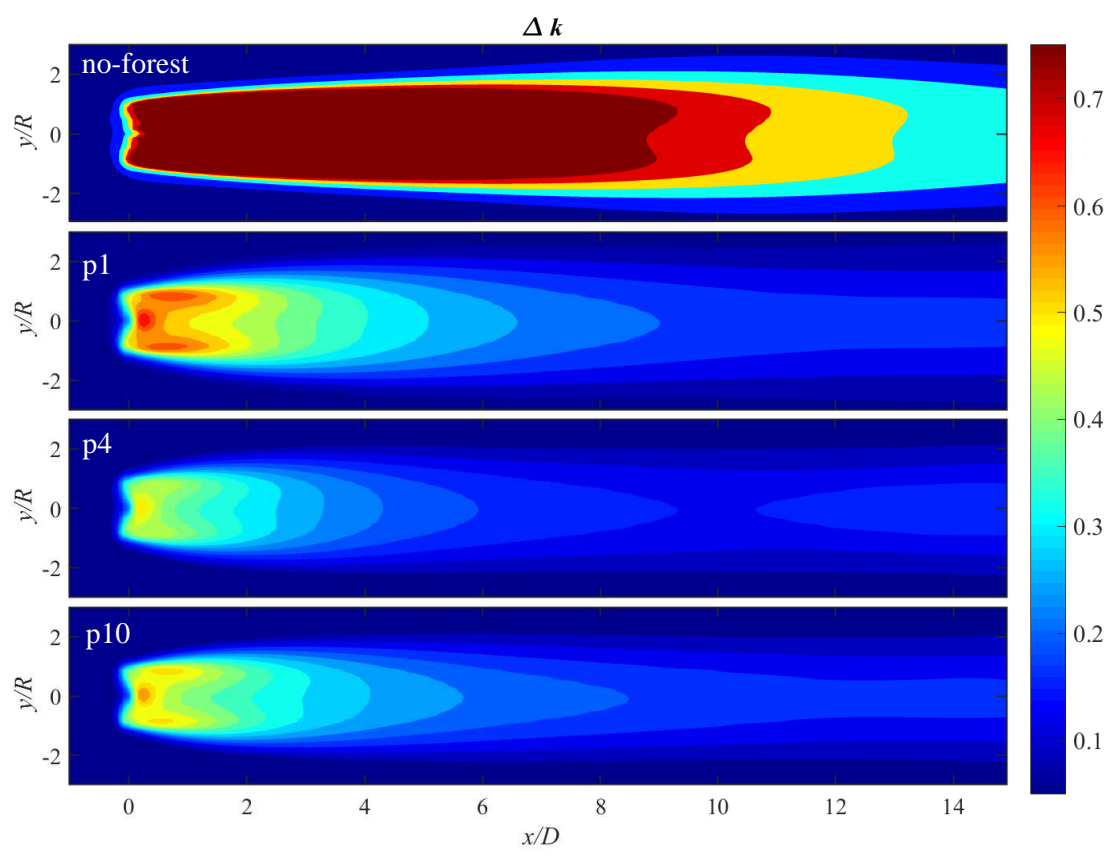

Figure 15: TKE increase $(\Delta k)$ contours on horizontal planes at the hub height.
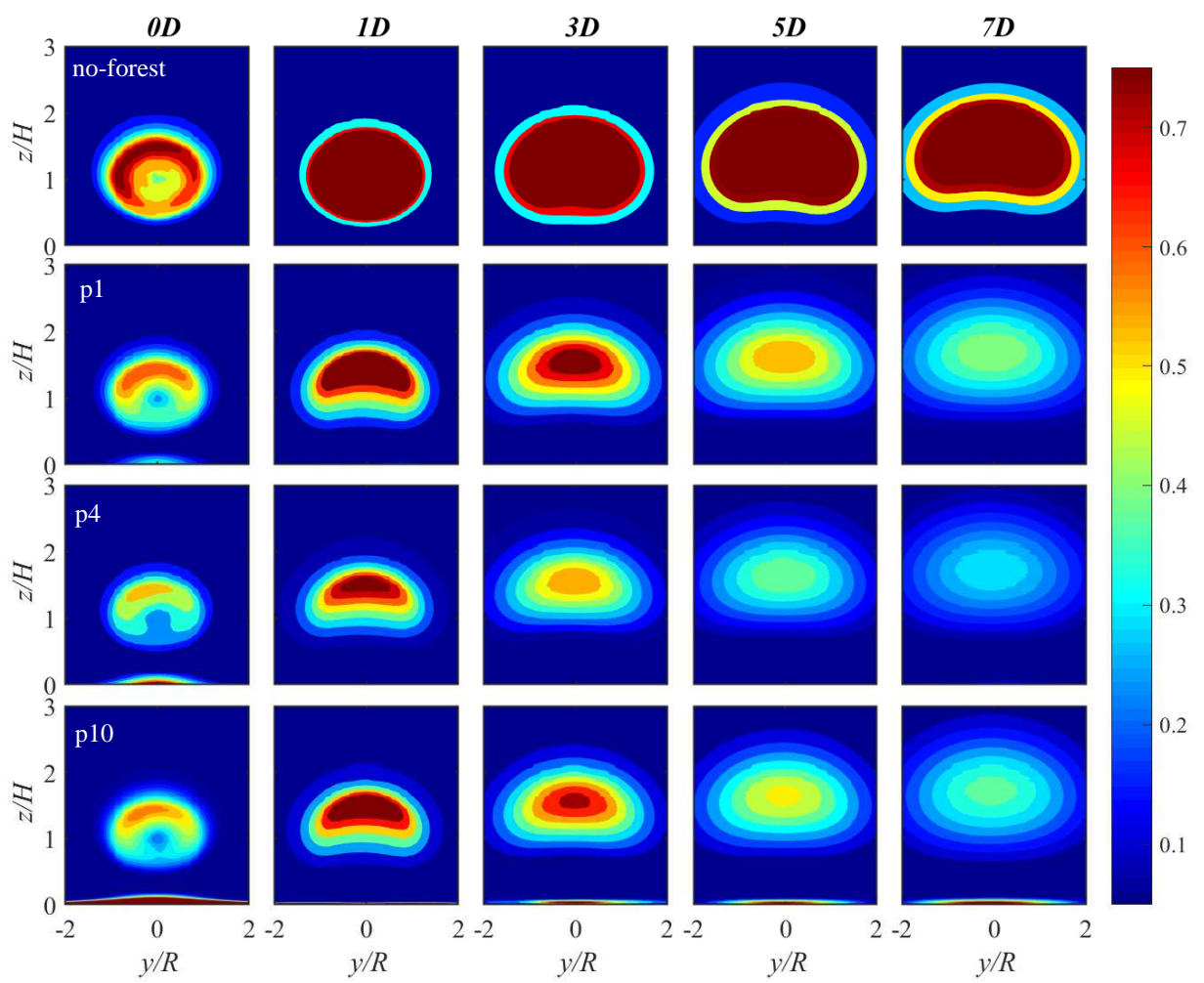

Figure 16: TKE-increase $(\Delta k)$ contours on vertical planes at five different locations downstream of turbine.

Figure 19 shows the velocity deficits and TKE increase plotted against LAI exactly at the hub height at locations at $x=3 D, 5 D$ and $7 D$ downstream for all four cases. These locations are appropriate to quantify wake development and recovery when considering a next turbine in a large wind-farm scenario. The physics of the wake flow is associated with the permeability level of the forest canopies, and this behaviour is earlier exhibited in the upstream TKE presented in Figure 10. In general, Figure 19 shows a similar wake behaviour in the forest cases with little differences among them, however, the variabilities among them do not linearly 

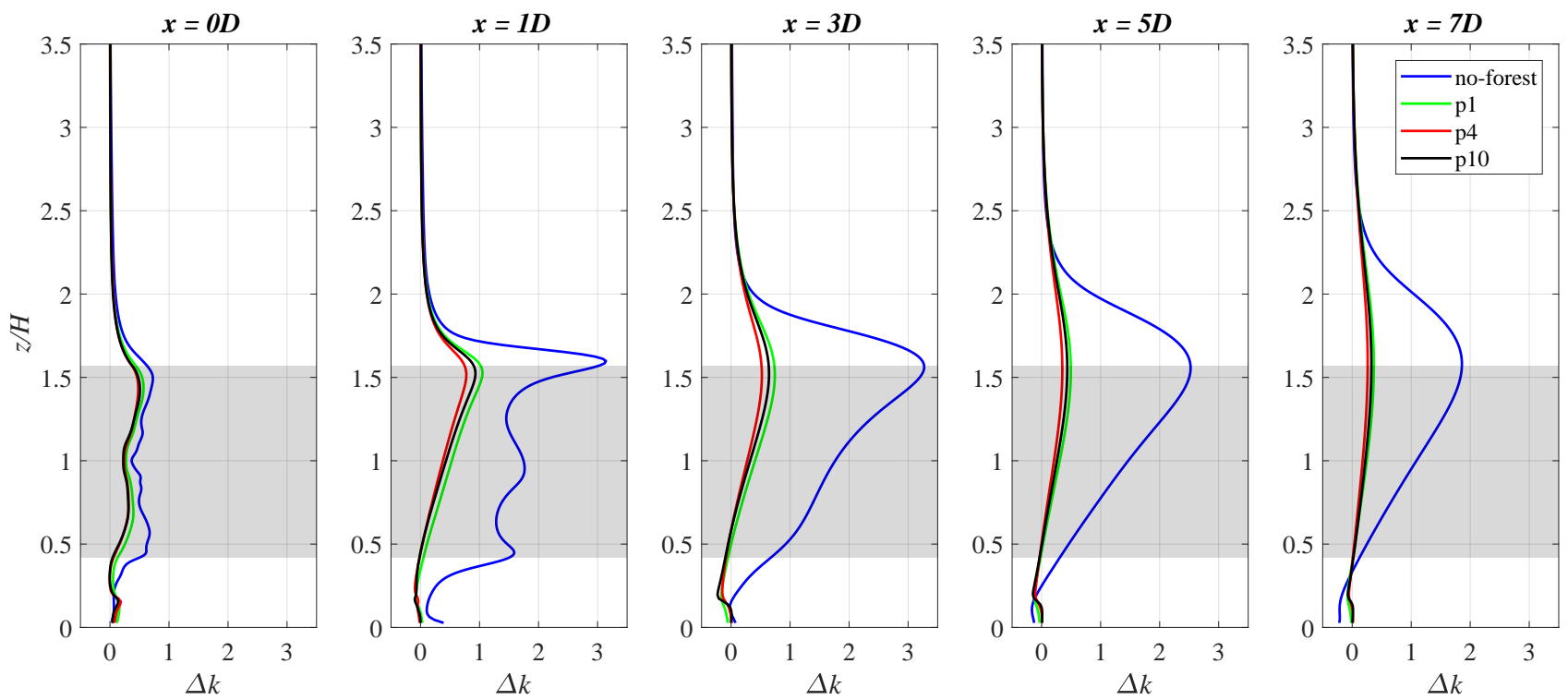

Figure 17: Vertical profiles of the TKE-increase $(\Delta k)$ at five different locations downstream of the turbine(The shaded region depicts the rotor area).
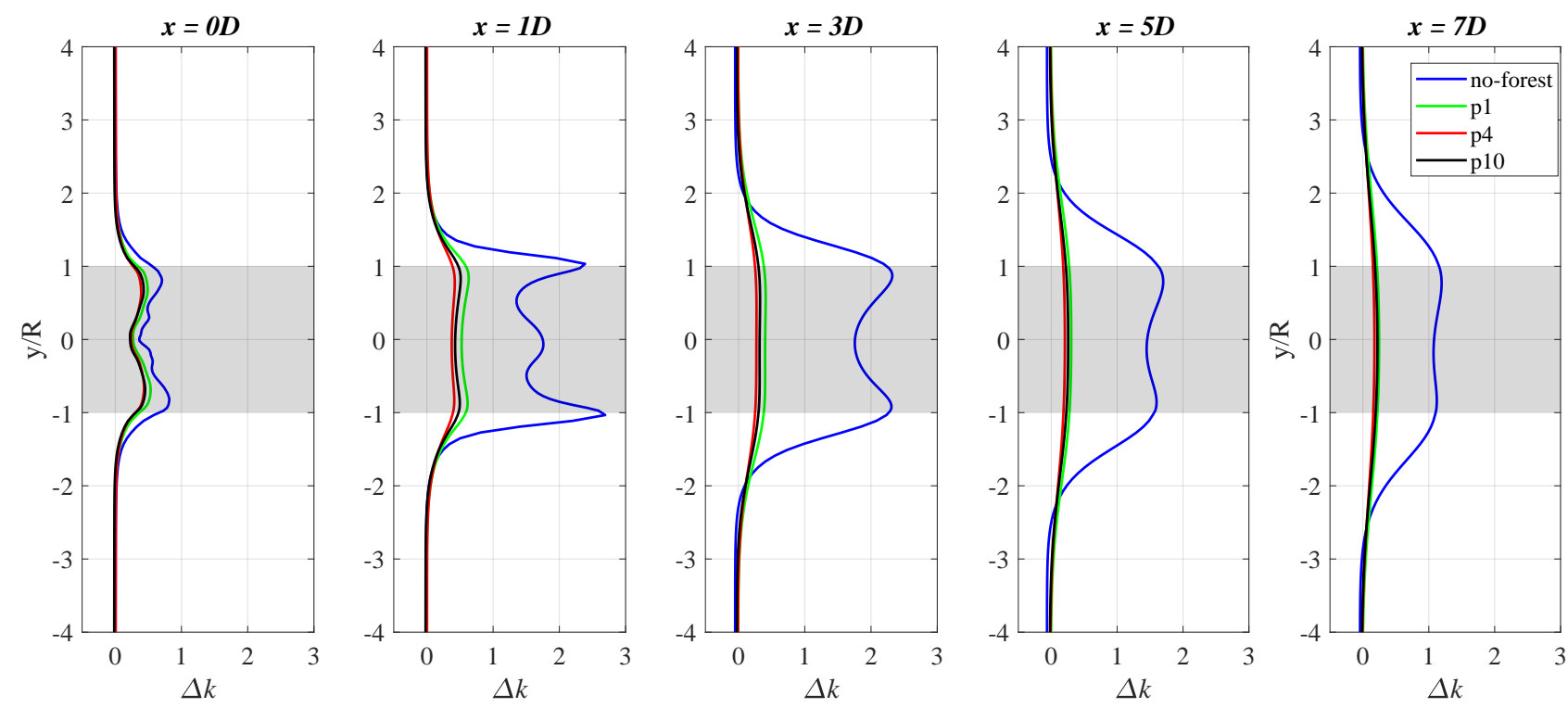

Figure 18: Horizontal profiles of the TKE-increase $(\Delta k)$ at five different locations downstream of the turbine (The shaded region depicts the rotor area).

depend on the permeability levels but rather on their upstream TKE level. The velocity-deficit and TKEincrease decrease from the no-forest case to $\mathrm{p} 1$ and to $\mathrm{p} 4$, which implies that the decreasing permeability (between $\mathrm{p} 1$ to $\mathrm{p} 4$ ) triggers the vertical flow fluctuations (and thus rising upstream TKE levels) which weakens the wake as the LAI increases. But from $\mathrm{p} 4$ to p10, an opposite behaviour is observed, where the wake begins to grow as although permeability continuously decreases, indicating a gradual development of the wake as the LAI increases (i.e. upstream TKE decreasing gradually from p5 to p10).

Figure 20 shows the wake development and recovery on a stream-wise line at the hub height. This figure helps to study the transition of the wake from the turbine location to the outlet. It is seen that the no-forest case exhibits a higher slope by $7.7 \%$ for $\Delta U$ and $70 \%$ for $\Delta k$ in the wake transition compared to the reference forest case (p10). Among the forest cases, the trend observed in the transition is maintained in a way that p1 and p10 are similar to each other while p4 is initially different from the rest (p1 and p10). The difference gradually vanishes and all three forest profiles overlap toward the outlet. 
(a)

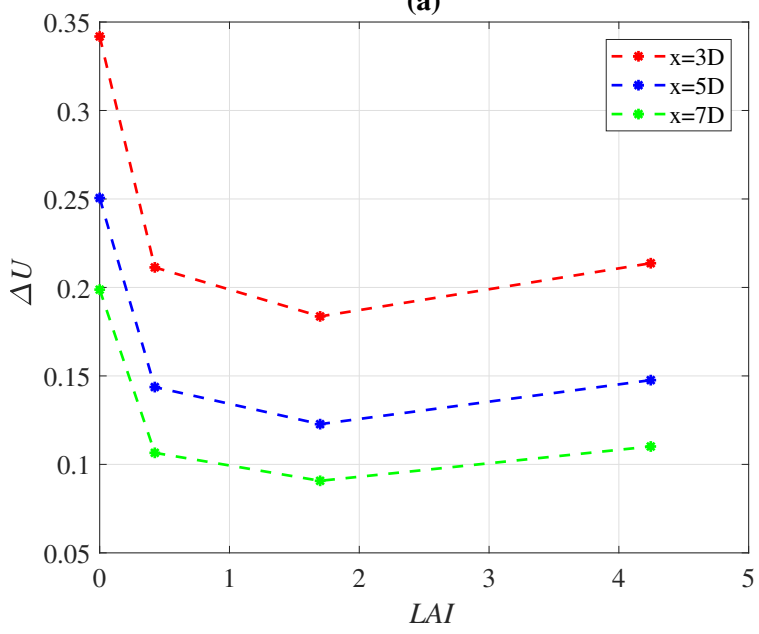

(b)

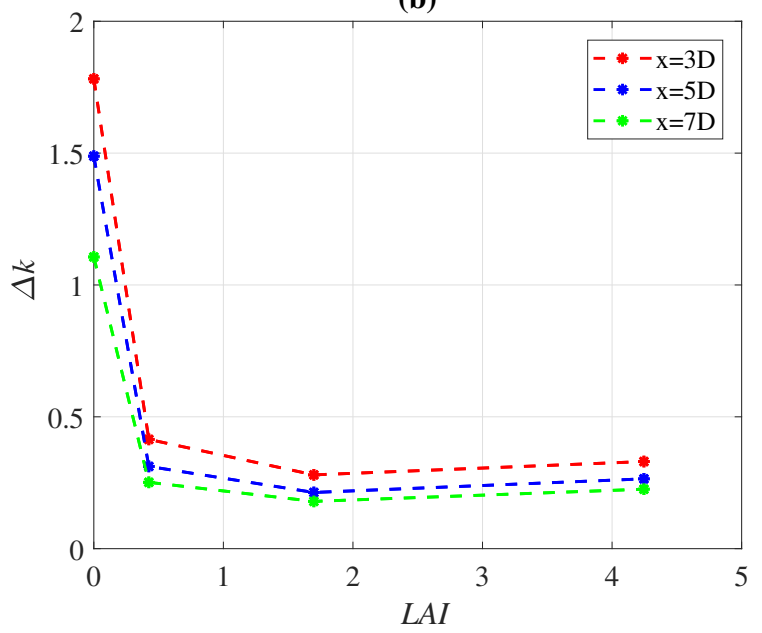

Figure 19: Plots of velocity-deficits (left) and TKE increase (right) against LAI values taken exactly at the turbine location.
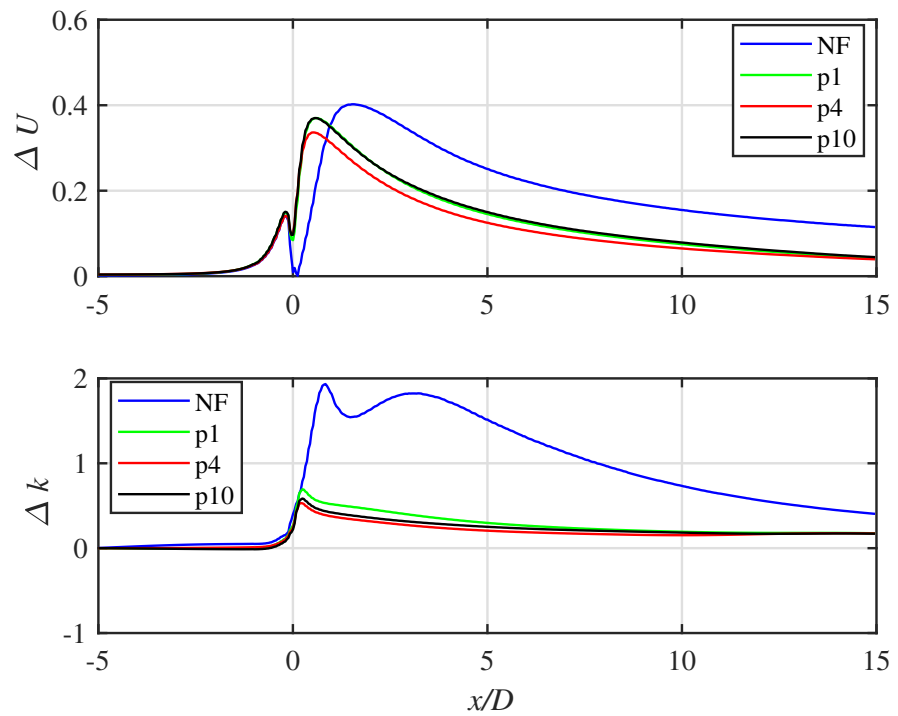

Figure 20: Velocity-deficits and TKE increase $(\Delta k)$ on horizontal planes at the hub height.

\section{Conclusions}

The paper presents an inspection of how different forest densities affect the ABL development and its subsequent effects on the wind-turbine wake. In total, 12 Pine forest cases and a no-forest case are considered in this study. The OpenFOAM software is utilized based on the RANS approach. The NREL 5MW windturbine is used as the reference turbine to study the wake. The turbine effects are modelled in the simulations using the ALM approach ${ }^{18}$. The simulations entail three-dimensional and two-dimensional computational domains for cases with turbine and without turbine respectively.

To assess the reliability of the present RANS simulations, a miniature wind-turbine wake is simulated, where the inflow profiles and wake characteristics are compared with the wind-tunnel experimental measurements of Chamorro and Porté-Agel ${ }^{41}$, reasonable agreement is observed between the present RANS results and the experimental data. A mesh dependency test is also carried out to ensure that the solution is independent of the chosen grid resolutions. The results from the reference forest simulations are validated against the field experimental data from a real forest site located at Ryningsnäs in the south-eastern Sweden ${ }^{32}$ and the results show a good agreement with the measurements.

Existing literature have shown that turbulence becomes more pronounced as canopy density increases, nonetheless, this current study has shown that this statement holds only for an increasing forest density of LAI up to an average of 1.7 , it afterwards decreases continuously as the density increases. On the other hand, the velocity within the canopy reduces with an increasing LAI. The velocity behaviour for LAI greater than 1.7 is similar above the hub height as the velocity increases with increasing LAI for LAI less than 1.7. 
Investigating the impact of forest density on the turbine wake, it is observed that the no-forest case has a clear distinction from all forest cases as it has the longest wake than the forest cases. The results indicate a huge damping of the TKE increase in the forest cases, which is highly beneficial for the next turbine (e.g. for wind-farm scenario) as the wake effects become weaker leading to a less disturbed approach of wind flow towards the second turbine. The forest with the highest upstream turbulence ( $\mathrm{p} 4$ with LAI $=1.70$ ) has the shortest wake and thus the fastest wake recovery, while the sparsest forest with LAI $=0.42$ and a dense forest ( $\mathrm{p} 10)$ with LAI $=4.25$ have a similar wake structure. In this perspective, turbines could be placed closer in wind farms with a similar forest density as p4. It is also observed that the velocity-deficit in the sparsest forest ( $\mathrm{p} 1$ with LAI $=0.42$ ) and the dense forest (p10 with LAI $=4.25$ ) behave similarly in terms of wake development and recovery although they both have significant differences in their velocities upstream.

Due to the RANS technique in use, the present study is limited to the investigation of ABL and windturbine wakes only. A better analysis of wind-turbine parameters, turbulence characteristics and power production in different forest densities can be done with LES. Also, a qualitative and quantitative analysis of an array of wind turbines with different forest densities, especially, for LAI below 1.7, will bring further advancement in wind energy applications. The authors aim to extend this present study in this respect in the near future.

\section{Acknowledgements}

The authors gratefully acknowledge the financial aid from the department of Computational Engineering, School of Engineering Science, Lappeenranta-Lahti University of Technology, Finland. We also appreciate CSC - IT Center for Science, Finland on whose platform the computations in this project were performed. Finally, the effort of all reviewers of this manuscript is well appreciated.

\section{References}

[1] Henrik Sundgaard Pedersen and Wiebke Langreder. Forest - added turbulence: A parametric study on turbulence intensity in and around forests. Journal of Physics: Conference Series, 75(1):012062, 2007.

[2] A. Chaudhari, B. Conan, S. Aubrun, and A. Hellsten. Numerical study of how stable stratification affects turbulence instabilities above a forest cover: application to wind energy. Journal of Physics: Conference Series, 753(3):11 pages, 2016. Article id: 032037.

[3] S. Wylie and S Watson. Cfd study of the performance of an operational wind farm and its impact on the local climate: Cfd sensitivity to forestry modelling. In EGU General Assembly Conference Abstracts, page 14068. EGU General Assembly Conference Abstracts (Vol. 15), apr 2013. Provided by the SAO/NASA Astrophysics Data System.

[4] Bastian Nebenführ and Lars Davidson. Large-eddy simulation study of thermally stratified canopy flow. Boundary-Layer Meteorology, 156(2):253-276, Aug 2015.

[5] Sylvain Dupont and Yves Brunet. Influence of foliar density profile on canopy flow: A large-eddy simulation study. Agricultural and Forest Meteorology, 148(6):976 - 990, 2008.

[6] B. Nebenführ and L. Davidson. Prediction of wind-turbine fatigue loads in forest regions based on turbulent les inflow fields. Wind Energy, 20(6):1003-1015, 2017.

[7] Cian James Desmond, Simon J. Watson, Sandrine Aubrun, Sergio Ávila, Philip Hancock, and Adam Sayer. A study on the inclusion of forest canopy morphology data in numerical simulations for the purpose of wind resource assessment. Journal of Wind Engineering and Industrial Aerodynamics, 126:24 $-37,2014$.

[8] Bai Yang, Michael R. Raupach, Roger H. Shaw, Kyaw Tha Paw U, and Andrew P. Morse. Large-eddy simulation of turbulent flow across a forest edge. part i: Flow statistics. Boundary-Layer Meteorology, 120(3):377-412, Sep 2006.

[9] Cian Desmond, Simon Watson, Christiane Montavon, and Jimmy Murphy. Modelling uncertainty in trans simulations of thermally stratified forest canopy flows for wind energy studies. Energies, 11(7):1703, 2018.

[10] Hong-Bing Su, Roger H. Shaw, and Kyaw Tha Paw U. Two-point correlation analysis of neutrally stratified flow within and above a forest from large-eddy simulation. Boundary-Layer Meteorology, 94(3):423-460, Mar 2000.

[11] Michael D. Novak, Jon S. Warland, Alberto L. Orchansky, Rick Ketler, and Steven Green. Wind tunnel and field measurements of turbulent flow in forests. part i: Uniformly thinned stands. Boundary-Layer Meteorology, 95(3):457-495, Jun 2000. 
[12] J. Huang, M. Cassiani, and J. D. Albertson. The effects of vegetation density on coherent turbulent structures within the canopy sublayer: A large-eddy simulation study. Boundary-Layer Meteorology, 133(2):253-275, Nov 2009.

[13] Oxana Agafonova, Anna Avramenko, Ashvinkumar Chaudhari, and Antti Hellsten. Effects of the canopy created velocity inflection in the wake development in a large wind turbine array. Journal of Physics: Conference Series, 753(3):10 pages, 2016. Article id: 032001.

[14] O Agafonova. A numerical study of forest influences on the atmospheric boundary layer and wind turbines. PhD thesis, Lappeenranta University of Technology, P O Box 20, FI-53851, Lappeenranta, Finland, 2017.

[15] A Clifton and Rozenn Wagner. Accounting for the effect of turbulence on wind turbine power curves. Journal of Physics: Conference Series, 524:012109, jun 2014.

[16] H. G. Weller, G. Tabor, H. Jasak, and C. Fureby. A tensorial approach to computational continuum mechanics using object-oriented techniques. Computers in Physics, 12(6):620-631, 1998.

[17] Jason Jonkman, Sandy Butterfield, Walter Musial, and George Scott. Definition of a 5-MW reference wind turbine for offshore system development,. Technical Report NREL/TP-500-38060, National Renewable Energy Lab. (NREL), Golden, CO 80401, 2009.

[18] J Sørensen and W. Shen. Numerical modeling of wind turbine wakes. J. Fluids Eng., 124(2):393-399, 2002.

[19] Tsan-Hsing Shih, William W. Liou, Aamir Shabbir, Zhigang Yang, and Jiang Zhu. A new $k-\epsilon$ eddy viscosity model for high reynolds number turbulent flows. Computers ES Fluids, 24(3):227 - 238, 1995.

[20] Roger H. Shaw and Ulrich Schumann. Large-eddy simulation of turbulent flow above and within a forest. Boundary-Layer Meteorology, 61(1):47-64, Oct 1992.

[21] Benoit Dalpé and Christian Masson. Numerical study of fully developed turbulent flow within and above a dense forest. Wind Energy, 11(5):503-515, 2008.

[22] A. Chaudhari, O. Agafonova, A. Hellsten, and J. Sorvari. Numerical study of the impact of atmospheric stratification on a wind-turbine performance. Journal of Physics: Conference Series, 854(1):11 pages, 2017. Article id: 012007.

[23] Luis A. Martínez-Tossas, Matthew J. Churchfield, and Stefano Leonardi. Large eddy simulations of the flow past wind turbines: actuator line and disk modeling. Wind Energy, 18(6):1047-1060, 2015.

[24] Fernando Porté-Agel, Hao Lu, and Yu-Ting Wu. A large-eddy simulation framework for wind energy applications. In Proceedings of the 5th international symposium on computational wind engineering (CWE2010), Chapel Hill, North Carolina, May 23-27 2010. Proceedings of the 5th international symposium on computational wind engineering (CWE2010).

[25] Matthew J Churchfield, Sang Lee, John Michalakes, and Patrick J Moriarty. A numerical study of the effects of atmospheric and wake turbulence on wind turbine dynamics. Journal of turbulence, (13):N14, 2012.

[26] Niels Troldborg, Jens N Sørensen, and Robert Mikkelsen. Actuator line simulation of wake of wind turbine operating in turbulent inflow. Journal of Physics: Conference Series, 75:012063, jul 2007.

[27] Mandar Tabib, Adil Rasheed, and Trond Kvamsdal. Les and rans simulation of onshore bessaker wind farm: analysing terrain and wake effects on wind farm performance. Journal of Physics: Conference Series, 625(1):012032, 2015.

[28] Mandar Tabib, M Salman Siddiqui, Adil Rasheed, and Trond Kvamsdal. Industrial scale turbine and associated wake development - comparison of rans based actuator line vs sliding mesh interface vs multiple reference frame method. Energy Procedia, 137:487-496, 2017.

[29] Peter Bachant, Anders Goude, and Martin Wosnik. Actuator line modeling of vertical-axis turbines. arXiv preprint arXiv:1605.01449, 2016.

[30] H Olivares-Espinosa, S-P Breton, C Masson, and L Dufresne. Turbulence characteristics in a free wake of an actuator disk: comparisons between a rotating and a non-rotating actuator disk in uniform inflow. Journal of Physics: Conference Series, 555(1):012081, 2014.

[31] Luca Lavaroni, Simon J Watson, Malcolm J Cook, and Mark R Dubal. A comparison of actuator disc and bem models in cfd simulations for the prediction of offshore wake losses. Journal of Physics: Conference Series, 524(1):012148, 2014. 
[32] Johan Arnqvist, Antonio Segalini, Ebba Dellwik, and Hans Bergström. Wind statistics from a forested landscape. Boundary-Layer Meteorology, 156(1):53-71, Jul 2015.

[33] H Bergström, H Alfredsson, J Arnqvist, I Carlén, E Dellwik, J Fransson, H Ganander, M Mohr, A Segalini, and Söderberg S. Wind power in forests: wind and effects on loads. Technical Report Elforsk AB, Technical University of Denmark (DTU), DTU, 2013.

[34] A Chaudhari, V Vuorinen, O Agafonova, A Hellsten, and J Hämäläinen. Large-eddy simulation for atmospheric boundary layer flows over complex terrains with applications in wind energy. In 11th World Congress on computational mechanics, WCCM, pages 5205-5216. 11th World Congress on computational mechanics (WCCM-2014), 2014.

[35] Victor Mendoza, Ashvinkumar Chaudhari, and Anders Goude. Performance and wake comparison of horizontal and vertical axis wind turbines under varying surface roughness conditions. Wind Energy, $22(4): 458-472,2019$.

[36] M.H. Baba-Ahmadi and G. Tabor. Inlet conditions for les using mapping and feedback control. Computers ES Fluids, 38(6):1299 - 1311, 2009.

[37] Ashvinkumar Chaudhari, Ville Vuorinen, Jari Hämäläinen, and Antti Hellsten. Large-eddy simulations for hill terrains: validation with wind-tunnel and field measurements. Computational and Applied Mathematics, 37 (2):2017 - 2038, 2018.

[38] Ashvinkumar Chaudhari, Antti Hellsten, and Jari Hämäläinen. Full-scale experimental validation of large-eddy simulation of wind flows over complex terrain: The bolund hill. Advances in Meteorology, 2016:14 pages, 2016. Article ID 9232759.

[39] O. Agafonova, A. Avramenko, A. Chaudhari, and A. Hellsten. The effects of the canopy created velocity inflection in the wake development. AIP Conference Proceedings, 1738(1):480082, 2016.

[40] Ashvinkumar Chaudhari. Large-eddy simulation of wind flows over complex terrains for wind energy applications. PhD thesis, Lappeenranta University of Technology, P O Box 20, FI-53851, Lappeenranta, FINLAND, 2014.

[41] Leonardo P. Chamorro and Fernando Porté-Agel. A wind-tunnel investigation of wind-turbine wakes: Boundary-layer turbulence effects. Boundary-Layer Meteorology, 132(1):129-149, Jul 2009.

[42] Yu-Ting Wu and Fernando Porté-Agel. Large-eddy simulation of wind-turbine wakes: Evaluation of turbine parametrisations. Boundary-Layer Meteorology, 138(3):345-366, Mar 2011.

[43] Michael J. Dwyer, Edward G. Patton, and Roger H. Shaw. Turbulent kinetic energy budgets from a large-eddy simulation of airflow above and within a forest canopy. Boundary-Layer Meteorology, 84(1):23-43, Jul 1997.

[44] Fernando Porté-Agel, Yu-Ting Wu, Hao Lu, and Robert J. Conzemius. Large-eddy simulation of atmospheric boundary layer flow through wind turbines and wind farms. Journal of Wind Engineering and Industrial Aerodynamics, 99(4):154 - 168, 2011. 